\title{
Mesenchymal Stem Cells Inhibit the Proliferation and Migration of Fibroblast-like Synoviocytes in Rheumatoid Arthritis via Exosome-mediated Delivery of miRNAs
}

\section{Liangyu Mi}

Shanxi Medical University

$\mathrm{Na}$ Li

Shanxi Medical University

Jinfang Gao

Shanxi Academy of Medical Sciences: Shanxi Bethune Hospital

\section{Xinyue Peng}

Shanxi Medical University

\section{$\mathrm{Na}$ Zhang}

Shanxi Academy of Medical Sciences: Shanxi Bethune Hospital

Rongxiu Huo

Shanxi Medical University

Ying Liu

Shanxi Academy of Medical Sciences: Shanxi Bethune Hospital

Maomao Fu

Taiyuan Central Hospital

\section{Liyun Zhang}

Shanxi Academy of Medical Sciences: Shanxi Bethune Hospital

KeXu ( $\nabla$ zhaoxuke@hotmail.com )

Shanxi Academy of Medical Sciences: Shanxi Bethune Hospital

\section{Research}

Keywords: Rheumatoid arthritis, Fibroblast-like synoviocytes, Human umbilical cord mesenchymal stem cells-derived Exosomes, miRNA, Proliferation, Migration

Posted Date: October 20th, 2021

DOl: https://doi.org/10.21203/rs.3.rs-965123/v1 
License: (c) (i) This work is licensed under a Creative Commons Attribution 4.0 International License. Read Full License 


\section{Abstract}

\section{Background}

Rheumatoid arthritis (RA) is a chronic autoimmune disease characterized by aggressive and symmetrical polyarthritis. Fibroblast-Like Synoviocytes(FLSs)play a central role in the pathogenesis of RA. The abnormal expression of miRNAs in RA FLSs mediates RA joint inflammationखsynovial hyperplasia囚and tissue destruction; MSC-Exos-derived miRNAs are a potential RA treatment strategy. This study aimed to investigate the hUCMSC-Exos can deliver miRNA to RA FLSs and affect their biological properties.

\section{Methods}

Primary hUCMSCs were isolated and cultured by tissue adherence method囚and surface markers were identified by flow cytometry. We used differential centrifugation combined with ultrafiltration to obtain

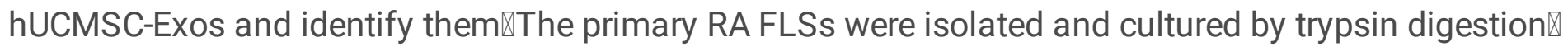
then surface markers were identified by flow cytometry. We labeled the RNA of hUCMSC-Exos. hUCMSCExos was co-cultured with RA FLSs then the dyed RA FLSs were observed with a fluorescence microscope.

GV493 with siRNA target sequences that could knock down Ago2 transfected hUCMSCs. Real-time PCR and Westen blot analysis Ago2 levels in hUCMSCs and transfected hUCMSCs to determine knockdown

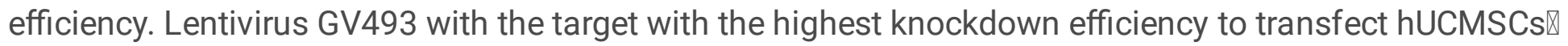
named hUCMSC ${ }^{\text {KD3-Ago2 }}$. Then hUCMSC-Exos $囚$ hUCMSC ${ }^{\text {KD3-Ago2 }}$-Exos $囚 h U C M S C{ }^{N C}$-Exos were extracted by the differential centrifugal method combined ultrafiltration method then were identified. The real-time cell analysis was used to detected the proliferation and migration of RA FLSs in different concentrations of hUCMSC-Exos. Finally $₫$ we used the optimal concentration of hUCMSC ${ }^{\text {KD3-Ago2 }}$-Exos and hUCMSC ${ }^{\mathrm{NC}}$ Exos to intervene in RA FLSs.

Results

After incubation with hUCMSC-Exos and RA FLSs》the hUCMSC-Exos accumulated in the blue nuclei of

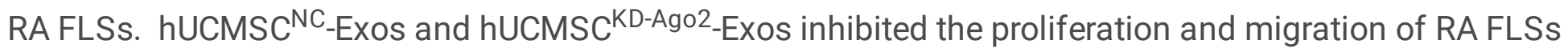

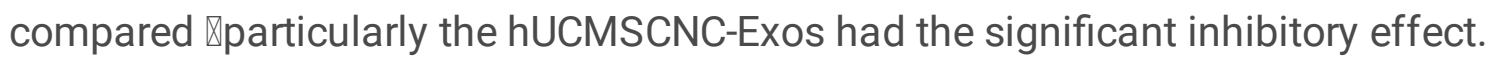

Conclusions

hUCMSC-Exos RNA can be taken up by RA FLSs $₫$ and hUCMSC may affect the proliferation and migration of RA FLSs via exosome-mediated delivery of miRNA.

\section{Highlights}

- hUCMSCs can deliver hUCMSC-Exos to RA FLSs. 
- Knockdown of Ago2 in hUCMSCs would reduce Ago2 in hUCMSC-Exos to affect miRNAs in hUCMSCExos.

- hUCMSC-Exos inhibits the proliferation and migration of RA FLSs.

- hUCMSC-Exos may affect RA FLSs by delivering miRNAs.

\section{Introduction}

Rheumatoid arthritis (RA) is an autoimmune disease characterized by the abnormal synovial hyperplasia of joints and the destruction of cartilage and bone. In recent years $囚$ the treatment strategies for RA have developed rapidly खbut there is still a long way to go before the complete cure of RA. Traditional NSAIDs are restricted by a variety of safety issues $₫$ while traditional DMARDs have a slow effect and serious side effects. Although the glucocorticoids can quickly eliminate joint swelling and reduce pain囚it cannot prevent joint damage and the development of lesions $₫$ moreover $\bigotimes i t$ also has potentially severe side effects after long-term use[1]. Although biological agents and molecular targeted drugs have improved remission rates in RA囚these drugs are expensive and target a particular step in the progression of RA.

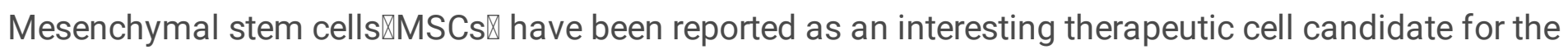
treatment of RA due to their immunomodulatory and differentiation effects. Unfortunately囚it also has been found to cause abnormal differentiation and tumor formation[2]. Patients with RA have a low treatment compliance rate and are prone to relapse after drug withdrawal. In short\none of the existing treatment methods for RA can enable patients to achieve long-term remission without drugsðand may even progress to persistent inflammation and progressive disability. Fibroblast-like synoviocytes (FLSs) are an important part of the synovial membrane $₫$ which play a significant role in the process of local

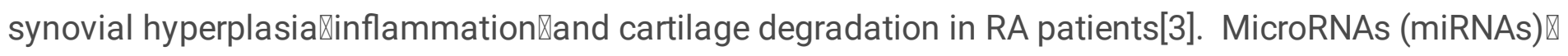

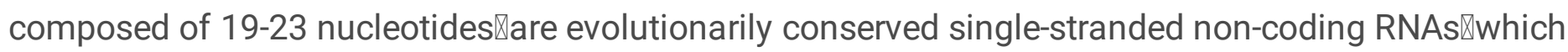
could negatively regulate protein expression by degrading target RNA or blocking its translation[4]. Existing studies have shown that miRNA in RA FLSs may participate in the pathological process of RA by

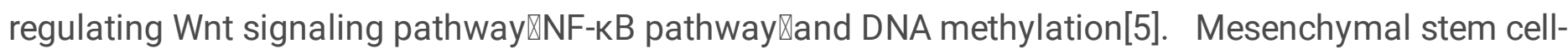
derived exosomes (MSC-Exos) are common membrane-bound nanovesicles with a size of about 30$150 \mathrm{~nm}$ derived from mesenchymal stem cells through exocytosis $₫$ which could mimic the function of

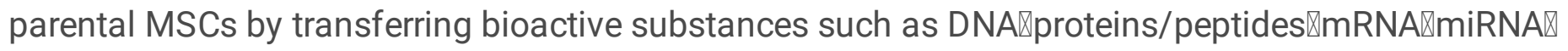
lipids\and organelles. MSC-Exos are more effective区less toxic \and more stable than the parental cells. Studies have shown that MSC-Exos can mediate intercellular communication and regulate different signaling pathways through miRNA transmission[6]. Thus $\rrbracket$ we have reason to believe the cell-free therapy based on MSC-Exos may be more effective in repairing the joint damage of RA囚breaking through the limitations of existing treatments囚and becoming a potential method for the treatment of RA. Excitingly囚the miRNAs encapsulated in MSC-Exos transfer information to recipient cells[7]. Some studies characterized the mechanisms by which Exos $₫$ particularly through miRNAs『are involved in RA. The human MSC-Exos that overexpress miRNA-124a could inhibit cell proliferation》migrationखand promote apoptosis in the FLSs line[8]. The bone marrow mesenchymal stem cell『BMMSC囚-secreted exosomal miR-192-5p can delay the event of the inflammatory response in RA[9]. BMMSC-derived Exos 
participate in the intercellular transfer of miR-320a and subsequently inhibit the progression of RA[10]. The DBA/1J mice BMMSC-derived miR-150-5p Exos decreased migration and invasion in RA FLSs \as well as $₫$ reduced hind paw thickness $\$ and the clinical arthritic scores in collagen-induced arthritis (CIA) mice[11]. Exos were extracted from normal MSCs with over-expressed miR-146a/miR-155 increased

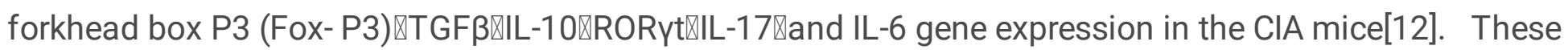
researches confirmed the value of MSC-Exos to mediate the direct intracellular transfer of miRNA and showed the efficacy of this strategy in RA.

The miRNAs have been found extracellularly $\bigotimes$ being encapsulated within Exos $₫$ or associated with Argonaute (Ago)[13].The mature miRNA is loaded into the RNA-induced silencing complex (RISC) $\varangle$ which mediates the relationship between the interacting miRNA and its target mRNA molecule. The Ago protein is part of RISC and mediates the cleavage of target mRNA. Particularly $\triangle A g o 2$ has splicing activity and can cut mRNA. Post-transcriptional Ago2 regulates miRNA expression abundance. Ago2 binds with miRNAs to form the RISC $₫$ which can associate with multivesicular bodies $₫$ which produce Exos after fusion with the plasma membrane[14]. In short\Ago2 promotes miRNA maturation囚prevents miRNA degradation\and controls the sorting of miRNA. Regulation of Ago2 could affect miRNA maturation.

In the current study $\otimes$ We tested the uptake of hUCMSC-Exos by RA FLSs and knocked down Ago2 in hUCMSC to reduce the content of Ago2 in hUCMSC-Exos $₫$ which is in order to the expression of miRNA in hUCMSC-Exos $₫$ then analyzed the effect of miRNA in hUCMSC-Exos on RA FLSs. In summary $\llbracket$ we clarified that miRNA delivered by hUCMSC-Exos may affect the proliferation and migration of RA FLSs. Ultimately囚the results provide a theoretical foundation for the application of hUCMSC-secreted exosomal miRNA in RA treatment.

\section{Materials And Methods}

\section{Ethics Statement}

Informed consent agreement forms were signed and collected from all participants before enrollment into our study. The research followed the tenets of the Declaration of Helsinki and was approved by the Ethical Committee of Shanxi Bethune Hospital (ethical approval code:2018LL007).

\section{Sample Collection}

The specimens of this study were obtained from patients in the Department of Orthopedics at Shanxi Bethune Hospital and the Second Hospital of Shanxi Medical University from March 2015 to March 2021. The synovial tissues $\bigotimes$ taken from arthroplasty or synovectomy were from patients with RA. All patients fulfilled the diagnosis of the American College of Rheumatology for RA.

\section{Isolation and Culture of RA FLSs}

The synovial tissues were collected $\llbracket$ cut under sterile conditions $₫$ detached with trypsin-

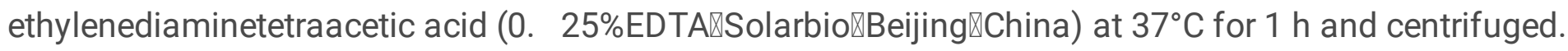


The cells were then collected and cultured in Dulbecco's modified Eagle's medium (DMEM)(GibcoखUSA)

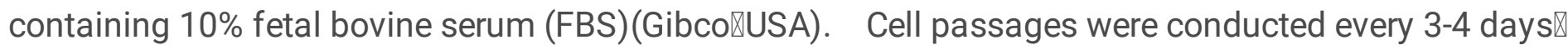
followed by $24-\mathrm{h}$ adherent growth of cells at $37^{\circ} \mathrm{C}$ with $5 \% \mathrm{CO} 2$. The cells were detached with $0.25 \%$ EDTA and passaged after reached $85 \%$ confluence. The RA-FLSs at passage 3 to 5 were utilized for the following experiments.

\section{Isolation and Culture of hUCMSCs}

We stripped the umbilical vein and artery of the umbilical cord (the Department of Obstetrics and Gynecology of Shanxi Bethune Hospital supplied) of the healthy term neonates. The remaining tissues were cut to $0.5 \mathrm{~mm} 3$ then inoculated in petri dishes. After the tissues were attached to the wall $\varangle$ we added serum-free medium (Excell BioهChain). The cells were fused to $80 \% \bigotimes$ they were digested by trypsin囚passed 1:3. The p3-p5 generation hUCMSCs in a good growth state were used in the experiment.

\section{Flow cytometry analysis}

RA FLSs cell suspension $\left(1 \times 10^{5}\right.$ cells $\left./ \mathrm{mL}\right)$ and hUCMSCs cell suspension $\left(1 \times 10^{5}\right.$ cells $\left./ \mathrm{mL}\right)$ were used for

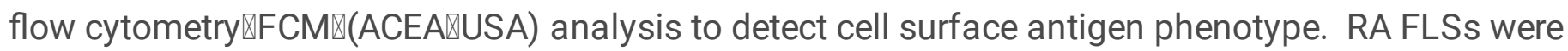
incubated on ice for $1 \mathrm{~h}$ with anti-rat antibodies (BioLegend囚USA) conjugated with fluorescein isothiocyanate(FITC) \Allophycocyanin(APC)『or phycoerythrin (PE). Then FCM evaluated and analyzed CDH 11 1 PDPN $\square C D 68$ (Abcam $₫ U S A)$. The hUCMSCs were incubated with anti-human monoclonal antibodies (BioLegend囚USA) conjugated with FITC or PE. The FCM evaluated and analyzed markers of hUCMSCs (CD105ロCD73 and CD90)『endothelial cells (CD31 and CD34) and hematopoietic cells (CD45) (Biolengd囚USA).

\section{Culturing of Hela cells}

Hela cells $\llbracket$ supplied by the Chinese Academy of Sciences as Western blot positive reference cells $₫$ were cultured at $37{ }^{\circ} \mathrm{C}$ in the incubator containing $\mathrm{CO} 2$ with a volume fraction of $5 \%$ and saturated humidity. The liquid was changed once every two days. When cells fused to $80 \% \bigotimes$ they were passed at a ratio of $1: 5$.

\section{Transfection of hUCMSCs with Lentivirus GV493}

hUCMSCs suspension was inoculated in 96 -well culture plates $\left(5 \times 10^{4}\right.$ cells/well) to ensure that the cell fusion rate was about $20 \%$ after 24 hours. Experimental groupings $₫$ dosage of GV 493 and transfection conditions are shown in Table 1. The cell culture medium was changed 16 hours after transfection. The fluorescence expression was observed under the fluorescence microscope (Olympus $₫$ Japan)after $72 \mathrm{~h}$.

\section{Transfection of hUCMSCs with lentivirus GV493 with or without siRNA target}


Because exosomal miRNA is associated with Ago2 $\varangle$ we had designed three different siRNA targets that can knock down Ago2】then respectively loaded them on GW493 to transfect hUCMSCs to knock down hUCMSCs Ago2 and exosomal miRNA. The hUCMSCs suspension was inoculated in a 6-well plate $\left(1 \times 10^{5}\right.$ cells/well) whose fusion rate was about $20 \%$ after being cultured for 24 hours. hUCMSCs were transfected by GV493 according to the previously established transfection conditions. According to the different types of lentiviruses transfected $\$ the experiment was divided into 4 groups $囚 \mathrm{hUCMSC} \mathrm{KD}^{\mathrm{K}}$-Ago2 group: LVPSC85384-1 transfected hUCMSCsðhUCMSC ${ }^{K D 2-A g o 2}$ group: LVPSC85385-11 transfected hUCMSCs $\llbracket$ hUCMSC ${ }^{\text {KD3-Ago2 }}$ group: LVPSC85386-11 transfected hUCMSCs $₫ N C$ group:the empty GV493 transfected hUCMSCs. The hUCMSCs in the KD group were named hUCMSC ${ }^{\text {KD-Ago2 }}$ which including hUCMSC ${ }^{K D 1-A g o 2} \llbracket$ hUCMSC ${ }^{K D 2-A g o 2} \llbracket$ and hUCMSC ${ }^{K D 3-A g o 2}$. At the same time邓those in the NC group were named hUCMSC ${ }^{\mathrm{NC}}$. The informations of group and virus are shown in Table 2. After 16 hours of transfection冈the cells in each well were collected and transferred to T25 culture flasks for continued cultivation. The fluorescence rate was the positive transfection rate邓which was observed under a fluorescence microscope 72 hours after transfection.

\section{Detect the levels of Ago2 in hUCMSCsDhUCMSC ${ }^{\mathrm{KD}-\mathrm{Ago2}}$ and $\mathrm{hUCMSC}{ }^{\mathrm{NC}}$}

\section{Real-time PCR analysis}

Total RNA was extracted and purified using Trizol reagent (Shanghai Pufei Biotech Co. खLtd. ) according to the manufacturer's instructions and RNA was precipitated using isopropanol. The RNA quality determined by the spectrophotometer was OD260/OD280: 1. 8-2. O囚and the RNA concentration was 18 $000-2 \varangle 000 \mu \mathrm{g} / \mathrm{mL}$. Glyceraldehyde-3-phosphate dehydrogenase (GAPDH) was used as the internal

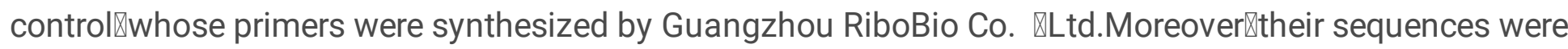
as follows: GAPDH-F5'-TGACTTCAACAGCGACACCCA-3' $₫$ R5'-CACCCTGTTGCTGTAGCCAAA-3'\Ago2F5'-TCCACCTAGACCCGACTTT-3' \R5'-GTTCCACGATTTCCCTGTT-3'. Reverse transcription of the extracted RNA was carried out according to the protocols of the M-MLV kit\Promega囚USA囚. Quantitative real-time PCR was performed using PCR Kit\QIAGEN『USA『on Real-time PCR instrument (Roche邓USA). With GAPDH used as the normalizing controls $\triangle \triangle C$ Ct indicates the gene expression level in cells $\llbracket$ and $2-\Delta \Delta C$ t reflects relative quantification.

\section{Isolation and identification of Exos}

We cultured hUCMSCs囚hUCMSC ${ }^{\mathrm{KD} 3-\mathrm{Ago} 2}$ and hUCMSC $^{\mathrm{NC}}$ with serum-free medium(Excell囚China) and separately collected supernatant\then we used ultrafiltration combined with differential centrifugation to obtain the corresponding exosomes. The process is shown in Figure 1.

Exos were visualized using transmission electron microscopy (JEM-2010 F囚JEOL囚Japan). The particle size of Exos was determined by highly sensitive nanoparticle size analysis and Nanoparticle Tracking Analysis (NTA) \using a Zetasizer NanoZS (Malvern Instruments囚Malvern囚UK). Exos protein concentrations were detected using a BCA Protein Assay kit (Solarbio $₫$ Beijing $\unrhd$ China). 


\section{Western blot analysis}

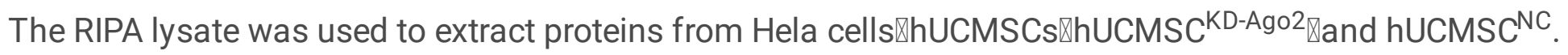
_ $40 \mu \mathrm{g}$ samples were separated with $10 \%$ sodium dodecyl sulfate-polyacrylamide gel electrophoresis gels \and transferred to polyvinylidene fluoride(PVDF) membrane『Millipore邓USA) after electrophoresis.

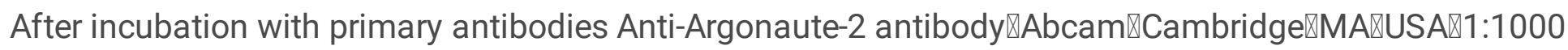
dilution囚in TBST with 5\% BSA囚the membranes were washed then incubated with goat anti-rabbit lgG-

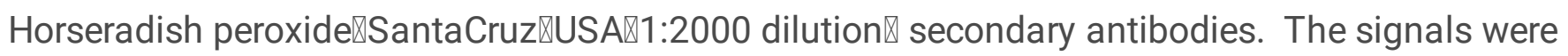
visualized with ECL chemiluminescent solution (Thermo FisherखUSA)

We used western blot to analyze the Exos surface antigen phenotype and Ago2 level. UCMSC-Exos』 hUCMSC ${ }^{\mathrm{KD} 3-A g o 2}$-Exos and hUCMSC ${ }^{\mathrm{NC}}$-Exos proteins were separated on $10 \%$ sodium dodecyl sulfatepolyacrylamide gel electrophoresis gels (SolarbioهChina)『and transferred to PVDF membrane after electrophoresis. PVDF membrane was blocked in TBST with $5 \%$ skim milk for $1 \mathrm{~h}$. After incubation with

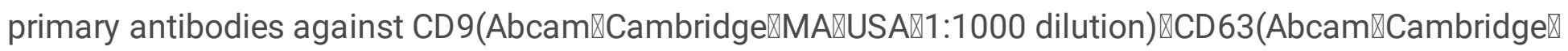

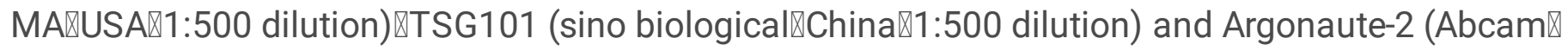
Cambridge区MA囚USA 1:1000 dilution) in TBST with 5\% BSA囚the membranes were washed four times with TBST (8 min each time) then incubated with horseradish peroxidase-conjugated goat anti-rabbit (CST区

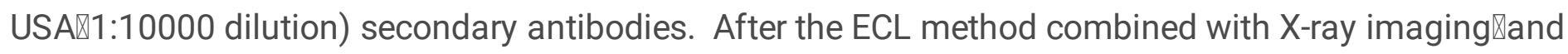
we analyzed the Ago2 level.

\section{Verification of the uptake of RNA in hUCMSC-Exos by RA FLSs}

According to the instructions $\llbracket$ we have configured RNA Select ${ }^{\text {TM }}$ Green Fluorescent Cell Stain (Thermo Fisher囚USA)working fluid冈then the working fluid was added to the prepared hUCMSC-Exos suspension to label RNA. Exosome Spin Columns(Thermo FisherखUSA)were used to remove residual dye from hUCMSCExos.

We set up control groups and hUCMSC-Exos intervention groups. First $\triangle 9 \mathrm{~mm}$ cell climbing sheets(NEST区

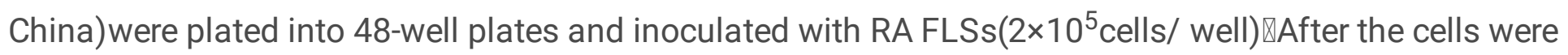
adherent囚the medium was changed. Complete medium was still added into the control wells冈and the UCMSC-Exos intervention wells were added with complete medium with $200 \mu \mathrm{g} / \mathrm{mL}$ RNA-labeled hUCMSC-Exos $₫$ then cells were incubated at $37^{\circ} \mathrm{C}$ in the dark for $24 \mathrm{~h}$. Next $₫$ the cells were washed with

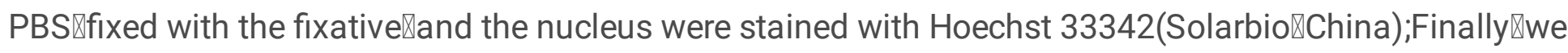
took out the cell climbing sheets and mounted them and fluorescence image were visualized with widefield high-content image analysis system(PerkinElmerखUSA).

\section{Analysis of the effect of hUCMSC-Exos on the proliferation and migration of RA FLSs}

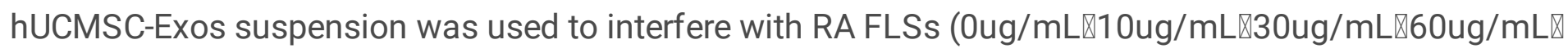
$90 \mathrm{ug} / \mathrm{mL} \otimes 120 \mathrm{ug} / \mathrm{mL} \otimes 150 \mathrm{ug} / \mathrm{mL} \otimes 180 \mathrm{ug} / \mathrm{mL}$ concentration);real-time Cell Analysis (RTCA)(ACEA囚 USA) was performed to detect Cell Index every $1 \mathrm{~h}$ to reflect the effect of hUCMSC-Exos on the 
proliferation and migration of RA FLSs. The RA FLSs suspension was inoculated into wells of E-Plate 16(ACEA囚USA) $\varangle\left(3 \times 10^{3}\right.$ cells/well). After the cells adhered冈the cell supernatant was replaced with the complete medium containing hUCMSC-Exos at different concentrations $\ a n d$ then the cells were cultured for 30 hours. Similarly $\mathbb{R T C A}$ was performed to detect the effect of hUCMSC-Exos on the migration of RA FLSs. The lower chambers of CIM-Plate 16(ACEA囚USA) were filled with 15\% FBS medium冈and the upper chamber was added with serum-free RA FLSs suspension containing different concentrations of hUCMSC-Exos (serum-free RA FLSs suspension containing different concentrations of hUCMSC-Exos (2. $5 \times 10^{4}$ cells/well) $\varangle$ then the cells were cultured for 48 hours.

\section{Assessment of hUCMSC-Exos miRNA on the proliferation and migration of RA FLSs}

We determined that the intervention concentration is $150 \mathrm{ug} / \mathrm{mL} \rrbracket$ which is the concentration that hUCMSCExos has the strongest effect on the proliferation of RA FLSs. RA FLSs were inoculated on E-Plate 16 $\left(6 \times 10^{3}\right.$ cells/well) for 48 hours. After the cells of each group adhered $\mathbb{2}$ we changed the culture conditions according to Table 3. RTCA detected Cell Index every 0 . 5h. RA FLSs were inoculated on CIM-Plate

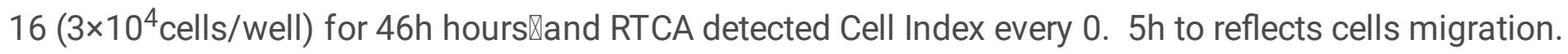
We constructed the RA FLSs culture system for each group of cells according to Table 4.

\section{Statistical analysis}

SAS 9. 4 is used for data processing and statistical analysis. Normally distributed data with homoscedasticity are expressed as mean \pm standard deviation $\Downarrow$ while the Non-normally distributed data are expressed as the interval between the median and quartile区and the qualitative data rate was expressed by the ratio. Statistical analysis: repeated measure ANOVA was used for normality; Non normality was compared by non-parametric tests based on rank. $P$ values less than 0.05 were considered statistically significant.

\section{Results}

\section{Isolation】culturingロand identification of RA FLSs}

RA FLSs grew by adhering to the substratum and displayed a fibroblast-like morphology (Figure 2a). Flow cytometry analysis of RA FLSs surface antigen phenotype revealed that PDPNGCDH-11 were expressed囚while CD68 were not. (Figure 2b).

\section{Isolation】culturingपand identification of hUCMSCs}

hUCMSCs grew by adhering to the substratum and displayed a long spindle-like morphology (Figure 3a).

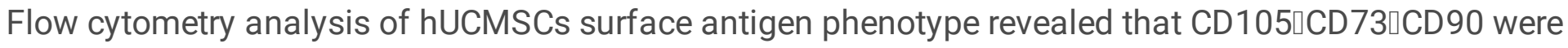

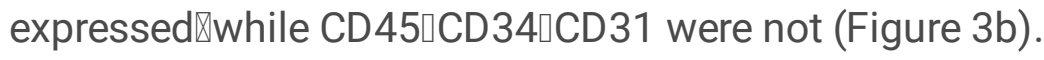


After GV493 was used to transfect hUCMSC under different conditions for 72 hours $囚$ the cells had normal growth morphology. GV493 is moderately difficult to transfect hUCMSCs囚and HiTransG P can significantly increase the transfection rate. The $80 \%$ fluorescence rate is most suitable between the $\mathrm{B}$ and $\mathrm{C}$ groups $\triangle \mathrm{Figure} 4 \mathrm{a} \bigotimes$. In other words $\bigotimes$ the corresponding $\mathrm{MOI}$ is between $10-50$. It follows that as few

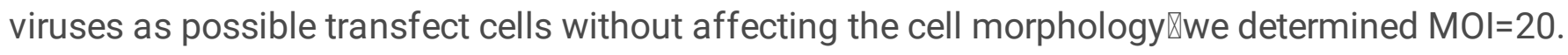

According to the most suitable transfection conditions that MOI=20 and add HiTransG P『we used GV493 with or without different targets transfected hUCMSCs. It showed that they successfully transfected hUCMSCs(Figure 4b).

\section{The Ago2 levels in hUCMSCsDhUCMSC ${ }^{\mathrm{KD}-\mathrm{Ago2}}$ and hUCMSC ${ }^{\mathrm{NC}}$}

\section{Real-time PCR analysis}

The larger the cell $\Delta \mathrm{Ct} \backslash \mathrm{t}$ the lower the target gene level. When the cell $\mathrm{t} \Delta \mathrm{Ct} \leq 12 \mathrm{Xth}$ the gene level of the cell

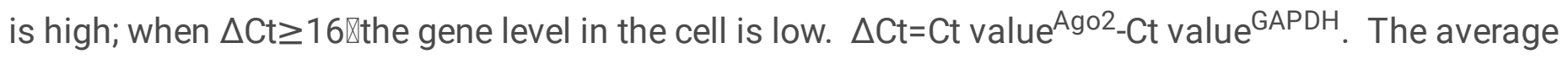
value of $\triangle \mathrm{Ct}$ in hUCMSCs is $8.91 \rrbracket$ so Ago2 is highly expressed in hUCMSCs. Amplification curve and dissolution curve of GAPDH and Ago2 of hUCMSCs $₫$ hUCMSC ${ }^{\mathrm{NC}}$ and hUCMSC ${ }^{\mathrm{KD}-A g o 2}$ are shown in Figure $5 a-f \llbracket Q$ uantitative PCR showed that compared with hUCMSC ${ }^{N C_{\text {the }}}$ tho 2 gene knockdown efficiency of

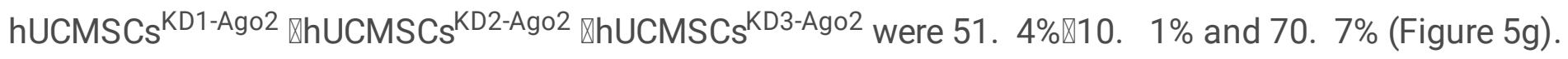

\section{Western blot analysis}

Western blot showing Ago2 expression in Hela cells (positive reference cells) 『which proves that the

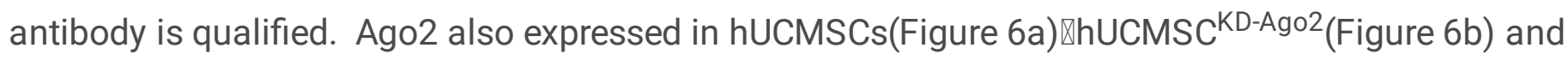
hUCMSC $^{\mathrm{NC}}$ (Figure $6 \mathrm{~b}$ ). The gray band values of hUCMSC ${ }^{\mathrm{NC}}$ are normalized囚hUCMSC ${ }^{\mathrm{KD} 1-}$

Ago2 hUCMSC $^{\mathrm{KD} 2-A g o 2}$ and hUCMSC ${ }^{\mathrm{KD} 3-A g o 2}$ band gray value ratios respectively are 0.84884713980 . 4890497ख0. 373544889.

All in all囚the results of real-time PCR and Western blot showed that the Ago2 knockdown rate was the highest in the hUCMSC ${ }^{\mathrm{KD} 3-\mathrm{AgO}} \llbracket$ so the corresponding target LVPSC85386-11 was selected for the experiment.

\section{Exos isolation and characterization}

Transmission electron microscopy revealed that hUCMSC-Exos $\llbracket$ hUCMSC ${ }^{\text {KD3-Ago2-Exos } ₫ \text { hUCMSC }}{ }^{\text {NC }}$ Exos were oval phospholipid bilayer-bound structures with diameters of 30-150 nm(Figure 7a). NTA showed that the diameter distribution of hUCMSC-Exos was a single peak》the median particle diameter was $146 \mathrm{~nm} \rrbracket$ and the concentration was $1.8 \times 10^{11}$ Particles/mL(Figure 7b). Highly sensitive nanoparticle size analysis showed that the particle size distributions of hUCMSC-Exos $₫$ hUCMSC ${ }^{\text {KD3-Ago2_ }}$ Exos $₫$ and hUCMSC ${ }^{\mathrm{NC}-E x o s}$ were single peaks $\ a n d$ their peak values respectively were $142 \mathrm{~nm} \rrbracket 106 \mathrm{~nm} \rrbracket$ $122 \mathrm{~nm}$ (Figure 7c). The protein concentrations of hUCMSC-Exos $₫$ hUCMSC ${ }^{\text {KD3-Ago2-Exos } ₫ a n d}$ 


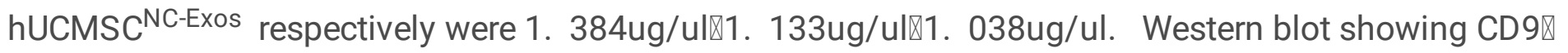

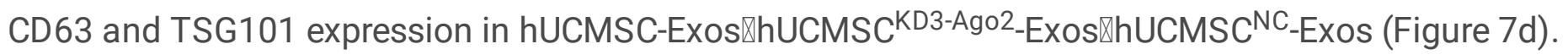
The level of Ago2 in hUCMSC ${ }^{\text {KD3-Ago2 }}$-Exos is lower than that in hUCMSC ${ }^{\mathrm{NC}-\mathrm{ExOS}_{S}}$ and hUCMSCExos(Figure 7e-f).

\section{The uptake of RNA in hUCMSC-Exos by RA FLSs}

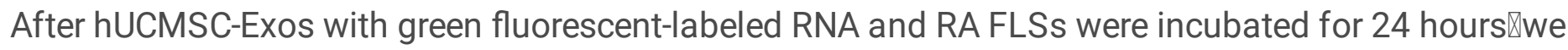
saw that the blue-stained nucleus of RA FLSs were accumulated by green fluorescence. It indicates that RA FLSs can uptake the RNA in hUCMSC-Exos $₫$ but compared with the control group $\$ the amount of cells in the experimental group is reduced (Figure 8a-b).

\section{The effect of hUCMSC-Exos on the proliferation and migration of RA FLSs}

After 6 hours of inoculationखall the RA FLSs adhered to the wall. At the same time『we added the complete medium containing hUCMSC-Exos $₫$ which caused a sharp bulge at the 6th hour of the curve. The cell index proliferation curve showed flattening after $25 \mathrm{~h}$. The $150 \mathrm{ug} / \mathrm{mL}$ hUCMSC-Exos inhibited the proliferation of RA FLSs most strongly(Figure 9a).

The proliferation of RA FLSs without hUCMSC-Exos intervention was slow\and we considered that it is related to fewer cells. In the subsequent experiments $₫$ the number of cells were increased to $6 \times 10^{3}$ cells/well . The migration curve showed that $120 \mathrm{ug} / \mathrm{mL}$ hUCMSC-Exos strongly inhibited the migration of RA FLSs (Figure 9b).

\section{The effect of hUCMSC-Exos miRNA on the proliferation and migration of RA FLSs.}

The proliferation curves and migration curves of RA FLSs reflect that hUCMSC ${ }^{\text {NC }}$-Exos and hUCMSC ${ }^{\text {KD3- }}$ Ago2-Exos inhibited the proliferation and migration of RA FLSs compared with the blank control group at

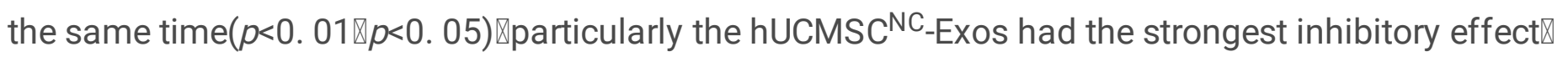
which. The proliferation and migration of RA FLSs in each group were statistically different in the Cell Index at different time points $(p<0.01 \rrbracket p<0.05)$ खand there were statistical differences between each group as a whole $(p<0.01 \rrbracket p<0.05)$ (Figure 10a-b). The proliferation and migration of RA FLSs at different time points in each group were expressed by cell index欧(Table 5a-b). We knocked down the Ago2 and also

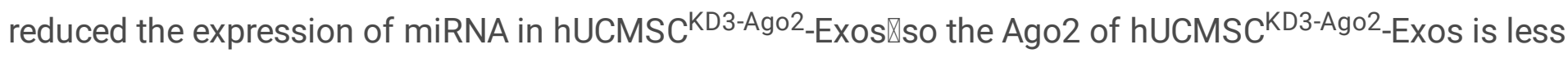
than hUCMSC ${ }^{N C}$-Exos. Those results indicates that miRNA in hUCMSC-Exos could affect the proliferation and migration of RA FLSs. It combines with that RA FLSs could take up the RNA of hUCMSC-Exos $₫$ which concludes hUCMSC-Exos may affect the proliferation and migration of RA FLSs by delivering miRNAs to RA FLSs. 


\section{Discussion}

MSCs have high proliferation囚immune regulation and multidirectional differentiation capabilities. they could inhibit the proliferation and differentiation of various immune cells『secretion of inflammatory factors and antibody production囚and promote the repair of damaged tissues. It has become a new method to explore the treatment of RA. We have proved that BMMSCs can inhibit The CXCL10/CXCR3 chemotactic axis adjusts the ratio of RANKL/OPG囚differentiates directly into chondrocytes $\ a n d$ improves the bone destruction of $\mathrm{CIA}[15]$.

MSCs were initially isolated from bone marrow and later found to be widely distributed in various tissues such as umbilical cord囚fat \synovium囚and gums. Compared with other sources囚UCMSCs have great differentiation potential『strong proliferation ability $\Downarrow$ low immunogenicity $\llbracket$ wide sources $\llbracket$ convenient materials $₫$ and no moral and ethical restrictions. In CIA rats $囚$ hUCMSCs inhibited the proliferation of T lymphocytes and promoted T lymphocyte apoptosis囚down-regulated RORyt mRNA and protein

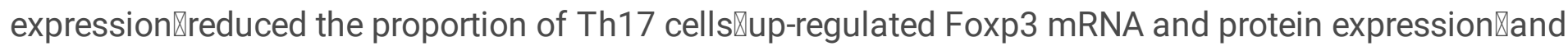
increased the proportion of spleen Treg cells. In addition区hUCMSCs down-regulated the expression of RORyt and Foxp3 in the joints囚inhibited IL-17 and promoted the expression of TGF-B in serum $\$ thereby improving arthritis \delaying radiological progress $₫$ and inhibiting synovial hyperplasia[16]. UCMSCs are most likely to become pluripotent stem cells with clinical application prospects. However囚UCMSCs have some problems in the treatment of RA. The survival and biological activity of UCMSCs after entering the body is not easy to control. Because MSCs treatment inhibits anti-tumor immune response邓there is a potential carcinogenic risk[17]. At the same time囚intravenous infusion of UCMSCs may cause capillary bed blockage区ectopic bone formationखcartilage formationखand tumor formation risks. Cell therapy of MSCs shows low implantation rate and poor survival. They play a role in animal models through paracrine[18][19] $₫$ in other words囚the protective effects of MSCs secretome depended on the presence of MSC-Exos[20].

Extracellular vesicles (EVs) released by MSCs in resting or stress state can mimic the immune regulation and tissue repair effects of MSCs $₫$ including Exos $₫$ microparticles (MPs) and apoptotic bodies[21]. Our previous research shows hUCMSC-EVs can improve CIA by simulating the immunomodulatory effects of

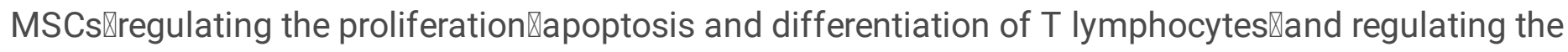
levels of inflammatory factors related to $\mathrm{T}$ lymphocytes[22]. As the most important componentखExos contains rich biological information delivery media such as mRNA and miRNA囚which can regulate the function『survival and homing of immune cells. Its immunomodulatory effect is stronger than MPs[23]区 and it has better properties as a non-cellular therapy than MSCs. hUCMSC-Exos are expected to become a new strategy for the treatment of RA.

Synovial hyperplasia can lead to irreversible cartilage and bone damage $₫$ which is the main pathological feature of RA and the difficulty in treatment. The Synovial lining layer is consisted of macrophage-like

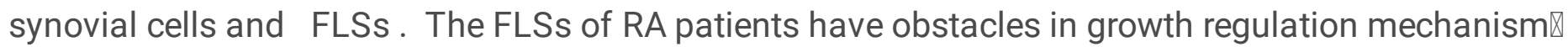
resulting in excessive proliferation of FLSs cells and showing "tumor-like growth"『which plays a central 
role in joint inflammation and cartilage degradation during the progression of RA. FLSs express vimentin $₫ C D 45 \rrbracket C D 55 \rrbracket C D 90 \varangle C D H 11 \rrbracket P D P N$ and other molecules on the surface. They can produce inflammatory factors and chemokines $\llbracket$ chemoattract immune cells to promote immune response and delay the resolution of inflammation[24]. RA FLSs may be a potential target for RA treatment.

Varieties of studies have reported that miRNAs in FLS can participate in the pathogenesis of RA by regulating multiple signaling pathways. MiRNAs could participate in the occurrence and development of diseases by blocking mRNA translation and then inhibiting protein expression. The Wnt signaling pathway is the first pathway worth mentioning. In RA FLSs $₫$ miRNAs participate in the pathogenesis of RA by regulating the Wnt signaling pathway. miR-26 increases the production of pro-inflammatory cytokines and promotes the apoptosis of RA FLSs[25]; miR-375 inhibits the production of matrix metalloproteinases and fibronectin[26]; the upregulation of miR-663 promotes the proliferation of RA FLSs[27]; miR-323-3P positively regulates the Wnt pathway to affect RA FLSs [28]. MiR-152 ectopic expression indirectly induces negative regulators of the Wnt pathway to inhibit FLS proliferation.It can regulate the Wnt signaling pathway at the level of ligand-receptor and intracellular factors to regulate a variety of proteins that promote the migration and differentiation of RA FLSs[29]. In addition》the miRNA in RA FLSs can also participate in the pathological progress of RA by regulating the NF-KB pathway. The up-regulated MiR-155 and miR-146a in RA FLSs inhibit the NF-KB pathway to promote the activation of RA FLSs and promote osteoclastogenesis[30][31] \miR-27a reduces the levels of MMP2 $\triangle M M P 9 \triangle M M P 13$ and Rho family proteins in RA FLSs[31] \thereby inhibiting the migration and invasion of RA FLSs『and blocking the NF-KB pathway of RA FLSs.In RA FLSs $₫$ miR-21 acts as an activator of the NF-KB pathway to promote NF$\mathrm{KB}$ nuclear translocation and promote the proliferation of RA FLSs [32]; miR-23b inhibits IL-17-induced activation of the NF-KB pathway and expression of inflammatory factors to inhibit the pathogenesis of RA [33]. miR-10a participates in the NF-KB-YY1-miR 10a pathway to promote the production of inflammatory factors and the proliferation and migration of RA FLSs [34]; miR-203 depends on the activity of NF-KB to regulate the expression of IL-6 in RA FLS [35]; miR-10a is expressed in RA FLSs》and inhibits the production of pro-inflammatory cytokines and matrix metalloproteinases by inhibiting NF-KB p65 induced by tumor necrosis factor-a; miR-18a directly acts on tumor necrosis factor-a-induced protein 3 to inhibit NF- KB pathway promotes the production of matrix-degrading enzymes and inflammatory mediators in RA FLS to promote the development of inflammation and cartilage destruction [36]; miR-19b positively regulates NF-KB signaling to promote inflammatory activation of RA FLSs [37]; DNA methylation and miRNAs in RA FLSs. The interaction between miR-124a is involved in the pathogenesis of RA. The methylation of miR-124a in RA FLSs promotes the proliferation of RA FLS and the expression of tumor necrosis factor-a[38]; ectopic expression of miR-152 down-regulates DNA methyltransferase DNMT1 and activates Wnt Pathway[29]; high expression of miR-34a*enhances the apoptosis of RA FLSs mediated by Fas-L and TRAIL[39].

The regulation of RA FLS by hUCMSC-Exos through miRNA will become a potential strategy for RA treatment. The amount of miRNA in Exos is extremely low $₫$ sequence analysis showed that approximately $13 \%$ of the total RNA content of EVs from milk is miRNAs[40]. So we labeled the RNA of hUCMSC-Exos and then co-incubated it with RA FLSs冈finally邓found that RA FLSs could take up RNA in 
hUCMSC-Exos. The RNA in hUCMSC-Exos may enter the RA FLSs directly through the surface protein and lipid ligands to directly activate the RA FLSs membrane surface receptors to generate signal complexes and activate the intracellular signaling pathway.It can also fuse with the plasma membrane of the RA FLSs or be endocytosed directly into the RA FLSs. The tracer methods for Exos include fluorescence

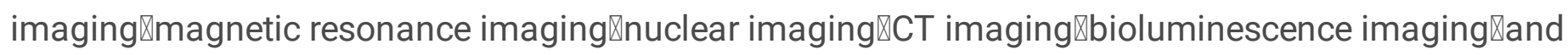
photoacoustic imaging[41] which help us to monitor Exos biological behavior in real-time and promote the development and delivery of targeted Exos in the field of diagnosis and medicine.

In addition $\nabla$ the co-incubation of hUCMSC-Exos and RA FLSs affected the proliferation and migration of RA FLSs. The $150 \mathrm{ug} / \mathrm{ml}$ hUCMSC-Exos strongly inhibited the proliferation of RA FLSs $₫$ and $120 \mathrm{ug} / \mathrm{ml}$ hUCMSC-Exos strongly inhibited the migration of RA FLSs. MSC-Exos can transport various biologically active proteins and RNA (mRNAðmiRNAØsiRNA囚etc. ) to target cells to perform their biological functions. MSCs-derived exosomal miRNA-124a[8]『miR-192-5p[9]『miR-320a[10]『miR-150-5p [11] and miR-

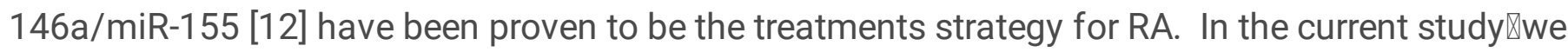
knocked down Ago2 in hUCMSC『and then the content of Ago2 in hUCMSC-Exos decreased. Ago2 participates in the sorting of miRNA in Exos $₫$ so the knockdown of Ago2 reduces the miRNAs expression exported by hUCMSC-Exos[14]. We used ago2 knockdown and non-knockdown hUCMSC-Exos to interfere with RA FLSs $₫$ respectively $\llbracket$ both of which inhibited the proliferation and migration of RA FLSs》especially the hUCMSC-Exos non-knockdown of ago2 had a stronger effect. The interfered miRNA in hUCMSC-Exos has a reduced effect on RA FLSs囚indicating that miRNA in hUCMSC-Exos affects the proliferation and migration of RA FLSs. Combining with that $\triangle R A$ FLSs could uptake the RNA of hUCMSC-Exos $₫$ we clarified that miRNA delivered by hUCMSC-Exos might affect the proliferation and migration of RA FLSs. In the future『we will use live cells to dynamically track hUCMSC-Exos in real-time to visualize the dynamic cellular uptake of Exos to provide a scientific basis for the interaction mechanism between Exos and cells. Not only that $\$ we will detect the changes of miRNAs in Exos and RA FLSs before and after Exos intervention in RA FLSs\and select more differentially expressed miRNAs to find more strategies for RA treatment.

\section{Conclusions}

hUCMSC-Exos may affect RA FLSs by delivering miRNAs. We traced that the RNA of hUCMSC-Exos was taken up by RA FLSs『and then knocked down the expression of hUCMSC-Exos Ago2】and confirmed that hUCMSC-Exos miRNA could inhibit the proliferation and migration of RA FLSs. Nextðwe will clarify the miRNA profile in RA FLSs $₫$ predict and anchor related therapeutic genes $\llbracket$ and use MSC-Exos-derived miRNAs to open new strategies for RA targeted therapy.

\section{Abbreviations}

RA:Rheumatoid arthritis; FLSs: Fibroblast-Like Synoviocytes; MSCs: Mesenchymal stem cells;miRNAs: MicroRNAs; MSC-Exos: Mesenchymal stem cell-derived exosomes; CIA:collagen-induced arthritis ; FoxP3:forkhead box P3;Ago: Argonaute ;RISC: RNA-induced silencing complex ; DMEM:Dulbecco's modified 
Eagle's medium; FBS:fetal bovine serum ;FCM:flow cytometry; FITC:fluorescein isothiocyanate; APC:Allophycocyanin;PE:phycoerythrin; GAPDH:Glyceraldehyde-3-phosphate dehydrogenase ; NTA:Nanoparticle Tracking Analysis ;FGF:Fibroblast growth factor;PVDF:polyvinylidene fluoride;RTCA:real-time Cell Analysis .

\section{Declarations}

\section{Authors' contributions}

KX directed the research.GLZ\NZ\YL and LYM designed the research $\triangle$ LYM and NL performed the

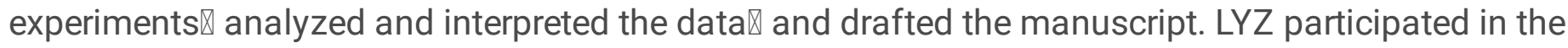
design and revised the manuscript. LYM $\triangle X Y P \otimes R X H$ and MMF performed the experiments. All authors have read and approved the final manuscript.

\section{Acknowledgements}

The authors thank the Translational Medicine Research Center of Shanxi Medical University for the excellent technical assistance.

\section{Funding}

This work was supported by grants from the National Natural Science Foundation (81871292) and the Key Research and Development Projects of Shanxi Province (201803D31136).

\section{Availability of data and materials}

The datasets used and/or analyzed during the current study are available from the corresponding author on reasonable request. Ethics approval and consent to participate The study was approved by the ethical committee of Shanxi Bethune Hospital. All experimental procedures were performed according to the Institutional.

\section{Availability of data and materials}

The datasets used and/or analyzed during the current study are available from the corresponding author on reasonable request.

\section{Ethics approval and consent to participate}

The study was approved by the ethical committee of Shanxi Bethune Hospital. All experimental procedures were performed according to the Use Committee guidelines of Shanxi Medical University.

\section{Consent for publication}

Not applicable. 


\section{Competing interests}

The authors have no competing interests.

\section{References}

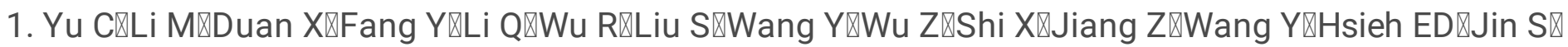

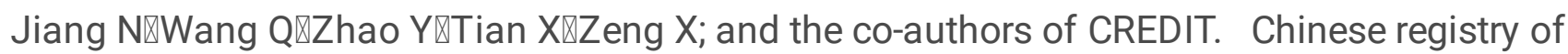
rheumatoid arthritis (CREDIT): I. Introduction and prevalence of remission in Chinese patients with rheumatoid arthritis. Clin Exp Rheumatol. 2018 Sep-Oct;36(5):836-840. Epub 2018 Mar 21.

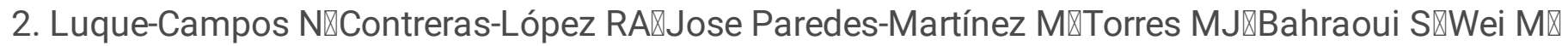
Espinoza F『Djouad F囚Elizondo-Vega RJ囚Luz-Crawford P. Mesenchymal Stem Cells Improve Rheumatoid Arthritis Progression by Controlling Memory T Cell Response. Front Immunol. 2019 Apr 16;10:798. doi: 10. 3389/fimmu. 2019. 00798. PMID: 31040848; PMCID: PMC6477064.

3. Nygaard G囚Firestein GS. Restoring synovial homeostasis in rheumatoid arthritis by targeting fibroblast-like synoviocytes. Nat Rev Rheumatol. 2020 Jun;16(6):316-333. doi: 10. 1038/s41584020-0413-5. Epub 2020 May 11. PMID: 32393826; PMCID: PMC7987137.

4. Wang L囚Liu LZ囚Jiang BH. Dysregulation of microRNAs in metal-induced angiogenesis and carcinogenesis. Semin Cancer Biol. 2021 Aug 21:S1044-579X(21)00221-2. doi: 10. 1016/j. semcancer. 2021. 08. 009. Epub ahead of print. PMID: 34428550.

5. Hong $W \llbracket Z$ hang $P \unrhd W a n g X \unrhd T u$ J\Wei W. The Effects of MicroRNAs on Key Signalling Pathways and Epigenetic Modification in Fibroblast-Like Synoviocytes of Rheumatoid Arthritis. Mediators Inflamm. 2018 May 10;2018:9013124. doi: 10. 1155/2018/9013124. PMID: 29861659; PMCID: PMC5971246.

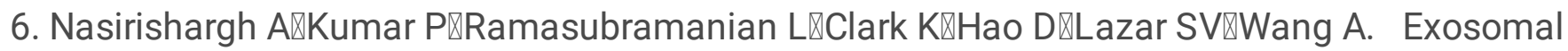
microRNAs from mesenchymal stem/stromal cells: Biology and applications in neuroprotection. World J Stem Cells. 2021 Jul 26;13(7):776-794. doi: 10. 4252/wjsc. v13. i7. 776. PMID: 34367477; PMCID: PMC8316862.

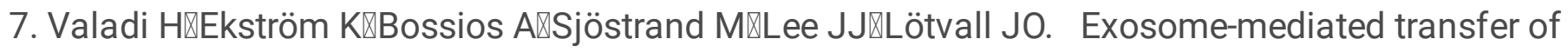
mRNAs and microRNAs is a novel mechanism of genetic exchange between cells. Nat Cell Biol. 2007 Jun;9(6):654-9. doi: 10. 1038/ncb1596. Epub 2007 May 7. PMID: 17486113.

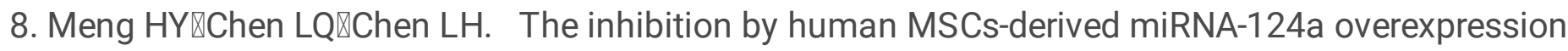
exosomes in the proliferation and migration of rheumatoid arthritis-related fibroblast-like synoviocyte cell. BMC Musculoskelet Disord. 2020 Mar 6;21(1):150. doi: 10. 1186/s12891-020-3159-y. PMID: 32143603; PMCID: PMC7060528.

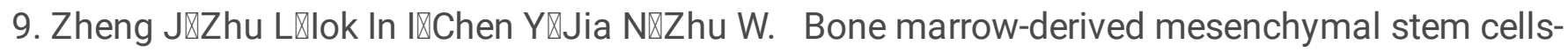
secreted exosomal microRNA-192-5p delays inflammatory response in rheumatoid arthritis. Int Immunopharmacol. 2020 Jan;78:105985. doi: 10. 1016/j. intimp. 2019. 105985. Epub 2019 Nov 24. PMID: 31776092. 
10. Meng Q®Qiu B. Exosomal MicroRNA-320a Derived From Mesenchymal Stem Cells Regulates Rheumatoid Arthritis Fibroblast-Like Synoviocyte Activation by Suppressing CXCL9 Expression. Front Physiol. 2020 May 26;11:441. doi: 10. 3389/fphys. 2020. 00441. PMID: 32528301; PMCID: PMC7264418.

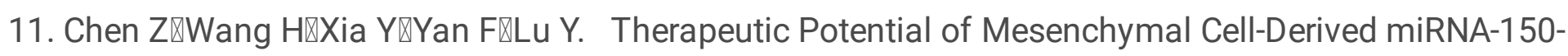
5p-Expressing Exosomes in Rheumatoid Arthritis Mediated by the Modulation of MMP14 and VEGF. J Immunol. 2018 Oct 15;201(8):2472-2482. doi: 10. 4049/jimmunol. 1800304. Epub 2018 Sep 17. PMID: 30224512; PMCID: PMC6176104.

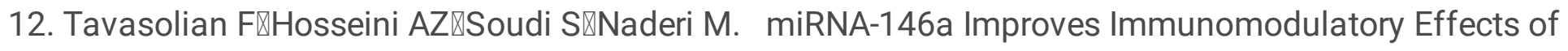
MSC-derived Exosomes in Rheumatoid Arthritis. Curr Gene Ther. 2020;20(4):297-312. doi: 10. 2174/1566523220666200916120708. PMID: 32938348.

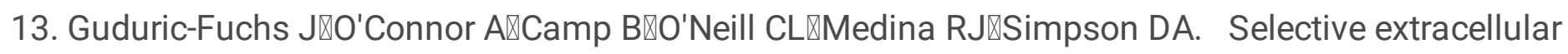
vesicle-mediated export of an overlapping set of microRNAs from multiple cell types. BMC Genomics. 2012 Aug 1;13:357. doi: 10. 1186/1471-2164-13-357. PMID: 22849433; PMCID: PMC3532190.

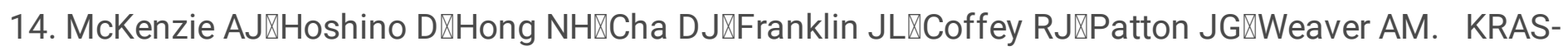
MEK Signaling Controls Ago2 Sorting into Exosomes. Cell Rep. 2016 May 3;15(5):978-987. doi: 10. 1016/j. celrep. 2016. 03. 085. Epub 2016 Apr 21. PMID: 27117408; PMCID: PMC4857875.

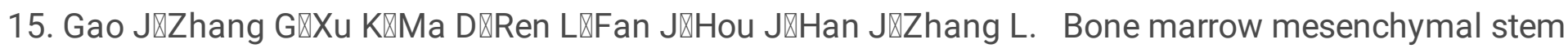
cells improve bone erosion in collagen-induced arthritis by inhibiting osteoclasia-related factors and differentiating into chondrocytes. Stem Cell Res Ther. 2020 May 7;11(1):171. doi: 10. 1186/s13287-020-01684-w. PMID: 32381074; PMCID: PMC7203805.

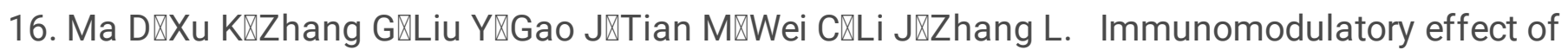
human umbilical cord mesenchymal stem cells on T lymphocytes in rheumatoid arthritis. Int Immunopharmacol. 2019 Sep;74:105687. doi: 10. 1016/j. intimp. 2019. 105687. Epub 2019 Jul 8. PMID: 31295689.

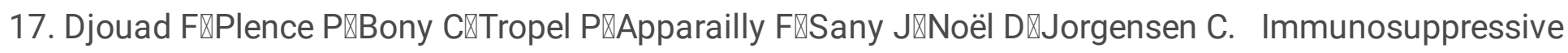
effect of mesenchymal stem cells favors tumor growth in allogeneic animals. Blood. $2003 \mathrm{Nov}$ 15;102(10):3837-44. doi: 10. 1182/blood-2003-04-1193. Epub 2003 Jul 24. PMID: 12881305.

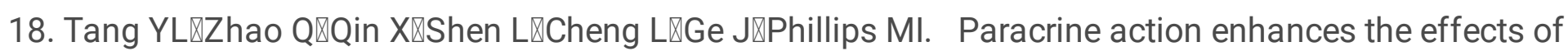
autologous mesenchymal stem cell transplantation on vascular regeneration in rat model of myocardial infarction. Ann Thorac Surg. 2005 Jul;80(1):229-36; discussion 236-7. doi: 10. 1016/j. athoracsur. 2005. 02. 072. PMID: 15975372.

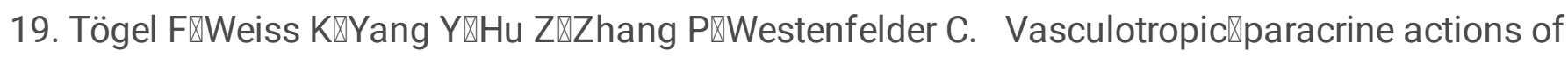
infused mesenchymal stem cells are important to the recovery from acute kidney injury. Am J Physiol Renal Physiol. 2007 May;292(5):F1626-35. doi: 10. 1152/ajprenal. 00339. 2006. Epub 2007 Jan 9. PMID: 17213465. 


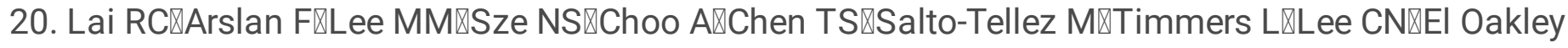
$\mathrm{RM} \otimes P a s t e r k a m p$ G『de Kleijn DP囚Lim SK. Exosome secreted by MSC reduces myocardial ischemia/reperfusion injury. Stem Cell Res. 2010 May;4(3):214-22. doi:10. 1016/j. scr. 2009. 12. 003. Epub 2010 Jan 4. PMID: 20138817.

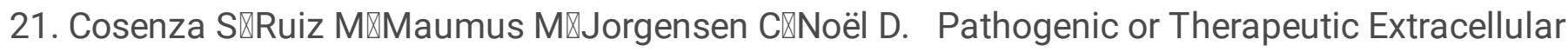
Vesicles in Rheumatic Diseases: Role of Mesenchymal Stem Cell-Derived Vesicles. Int J Mol Sci. 2017 Apr 22;18(4):889. doi: 10. 3390/ijms18040889. PMID: 28441721; PMCID: PMC5412468.

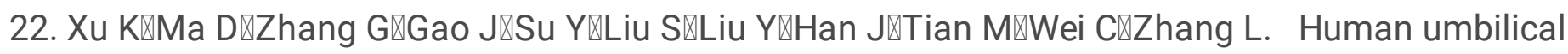
cord mesenchymal stem cell-derived small extracellular vesicles ameliorate collagen-induced arthritis via immunomodulatory T lymphocytes. Mol Immunol. 2021 Jul;135:36-44. doi: 10. 1016/j. molimm. 2021. 04. 001. Epub 2021 Apr 12. PMID: 33857817.

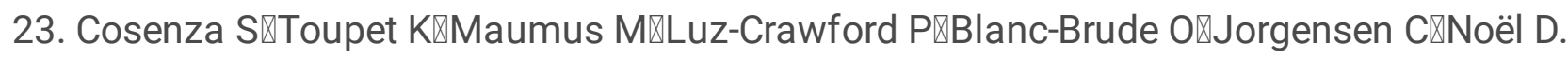
Mesenchymal stem cells-derived exosomes are more immunosuppressive than microparticles in inflammatory arthritis. Theranostics. 2018 Feb 3;8(5):1399-1410. doi: 10. 7150/thno. 21072. PMID: 29507629; PMCID: PMC5835945.

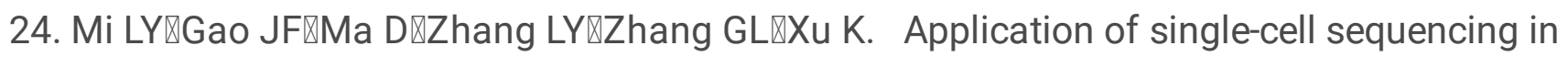
autoimmune diseases. Chin Med J (Engl). 2020 Sep 1;134(4):495-497. doi: 10. 1097/CM9. 0000000000001050 . PMID: 32889911; PMCID: PMC7909166.

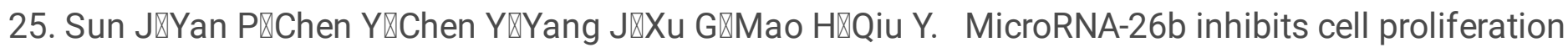

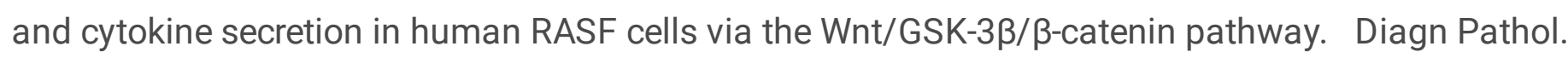
2015 Jun 19;10:72. doi: 10. 1186/s13000-015-0309-x. PMID: 26088648; PMCID: PMC4472173.

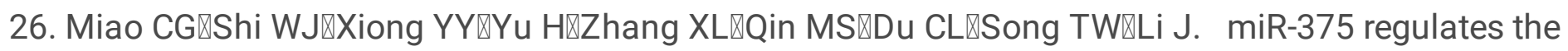
canonical Wnt pathway through FZD8 silencing in arthritis synovial fibroblasts. Immunol Lett. 2015 Mar;164(1):1-10. doi: 10. 1016/j. imlet. 2015. 01. 003. Epub 2015 Jan 22. PMID: 25619565.

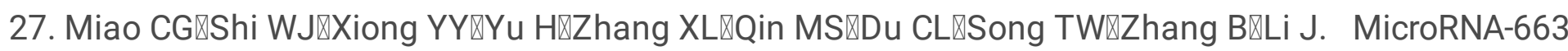
activates the canonical Wnt signaling through the adenomatous polyposis coli suppression. Immunol Lett. 2015 Jul;166(1):45-54. doi: 10. 1016/j. imlet. 2015. 05. 011. Epub 2015 May 28. PMID: 26028359.

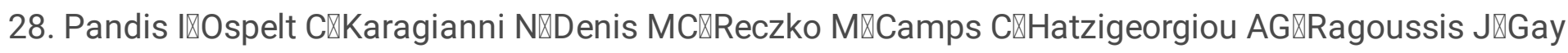
SøKollias G. Identification of microRNA-221/222 and microRNA-323-3p association with rheumatoid arthritis via predictions using the human tumour necrosis factor transgenic mouse model. Ann Rheum Dis. 2012 Oct;71(10):1716-23. doi: 10. 1136/annrheumdis-2011-200803. Epub 2012 May 5. PMID: 22562984.

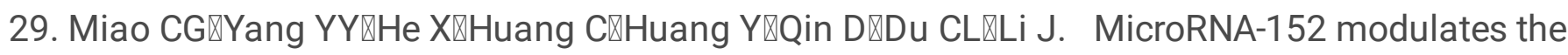
canonical Wnt pathway activation by targeting DNA methyltransferase 1 in arthritic rat model. Biochimie. 2014 Nov;106:149-56. doi: 10. 1016/j. biochi. 2014. 08. 016. Epub 2014 Sep 4. PMID: 25194984. 


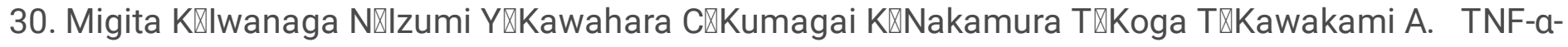
induced miR-155 regulates IL-6 signaling in rheumatoid synovial fibroblasts. BMC Res Notes. 2017 Aug 14;10(1):403. doi: 10. 1186/s13104-017-2715-5. PMID: 28807007; PMCID: PMC5556669.

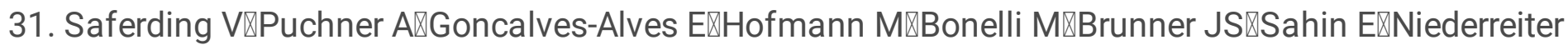

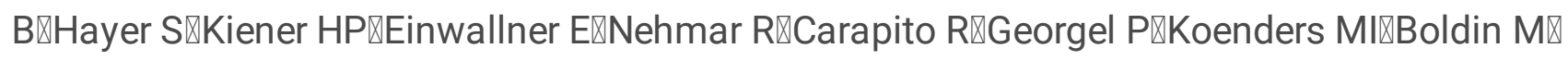

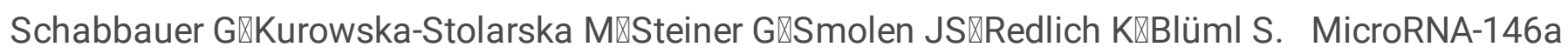
governs fibroblast activation and joint pathology in arthritis. J Autoimmun. 2017 Aug;82:74-84. doi: 10. 1016/j. jaut. 2017. 05. 006. Epub 2017 May 22. PMID: 28545737; PMCID: PMC7233286.

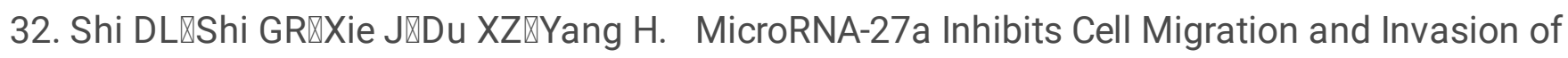
Fibroblast-Like Synoviocytes by Targeting Follistatin-Like Protein 1 in Rheumatoid Arthritis. Mol Cells. 2016 Aug 31;39(8):611-8. doi: 10. 14348/molcells. 2016. $0103 . \quad$ Epub 2016 Aug 8. PMID: 27498552; PMCID: PMC4990753.

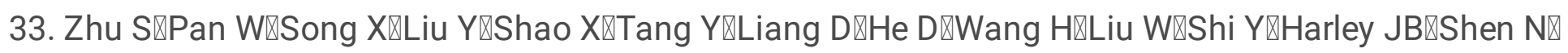
Qian Y. The microRNA miR-23b suppresses IL-17-associated autoimmune inflammation by targeting TAB2ХTAB3 and IKK-a. Nat Med. 2012 Jul;18(7):1077-86. doi: 10. 1038/nm. 2815. PMID: 22660635.

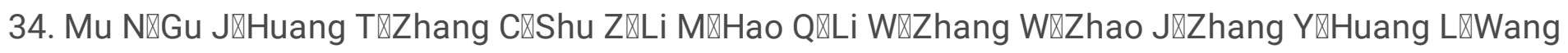

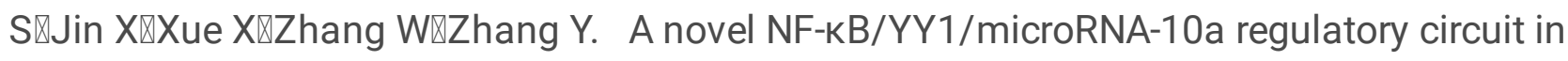
fibroblast-like synoviocytes regulates inflammation in rheumatoid arthritis. Sci Rep. 2016 Jan 29;6:20059. doi: 10. 1038/srep20059. PMID: 26821827; PMCID: PMC4731824.

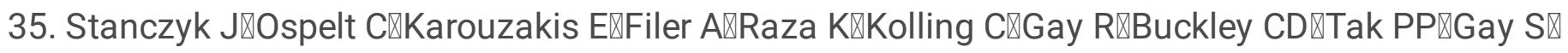
Kyburz D. Altered expression of microRNA-203 in rheumatoid arthritis synovial fibroblasts and its role in fibroblast activation. Arthritis Rheum. 2011 Feb;63(2):373-81. doi: 10. 1002/art. 30115. PMID: 21279994; PMCID: PMC3116142.

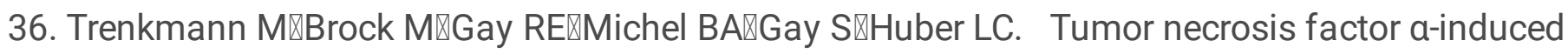
microRNA-18a activates rheumatoid arthritis synovial fibroblasts through a feedback loop in NF-KB signaling. Arthritis Rheum. 2013 Apr;65(4):916-27. doi: 10. 1002/art. 37834. PMID: 23280137.

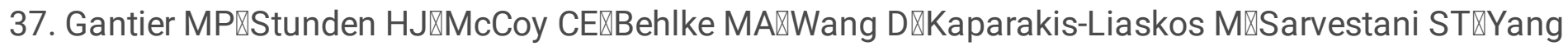

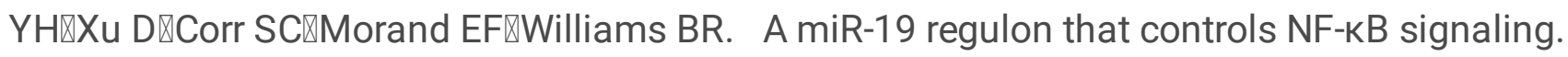
Nucleic Acids Res. 2012 Sep;40(16):8048-58. doi: 10. 1093/nar/gks521. Epub 2012 Jun 7. PMID: 22684508; PMCID: PMC3439911.

38. Zhou Q『Long L囚Zhou T囚Tian J『Zhou B. Demethylation of MicroRNA-124a Genes Attenuated Proliferation of Rheumatoid Arthritis Derived Fibroblast-Like Synoviocytes and Synthesis of Tumor Necrosis Factor-a. PLoS One. 2016 Nov 8;11(11):e0164207. doi: 10. 1371/journal. pone. 0164207. PMID: 27824863; PMCID: PMC5100945. 


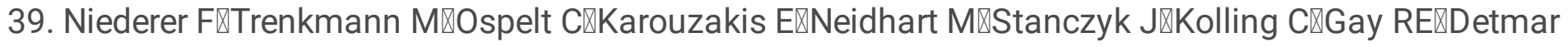
$M \varangle G$ ay $S \varangle$ Jüngel A $\varangle$ Kyburz D. Down-regulation of microRNA-34a* in rheumatoid arthritis synovial fibroblasts promotes apoptosis resistance. Arthritis Rheum. 2012 Jun;64(6):1771-9. doi: 10. 1002/art. 34334. Epub 2011 Dec 12. PMID: 22161761.

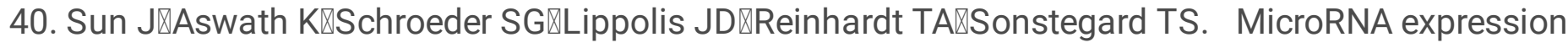
profiles of bovine milk exosomes in response to Staphylococcus aureus infection. BMC Genomics. 2015 Oct 16;16:806. doi: 10. 1186/s12864-015-2044-9. PMID: 26475455; PMCID: PMC4609085.

41. Li YJ $\llbracket W u ~ J Y \otimes W a n g ~ J M \otimes H u ~ X B \otimes X i a n g ~ D X$. Emerging strategies for labeling and tracking of extracellular vesicles. J Control Release. 2020 Dec 10;328:141-159. doi: 10. 1016/j. jconrel. 2020. 08. 056. Epub 2020 Aug 31. PMID: 32882270.

\section{Tables}




\begin{tabular}{|c|c|c|c|c|}
\hline Infection & A & B & C & Control \\
\hline \multicolumn{5}{|l|}{ Condition } \\
\hline \multicolumn{5}{|l|}{ GV493 } \\
\hline \multicolumn{5}{|l|}{ mount } \\
\hline \multicolumn{5}{|l|}{ QTUQ } \\
\hline \multirow{2}{*}{$\begin{array}{l}\text { Group A } \\
1 \times 10^{8}\end{array}$} & 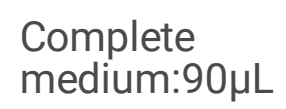 & 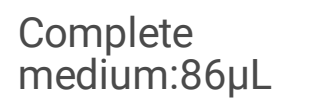 & 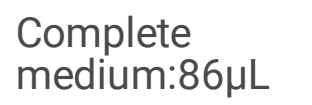 & $\begin{array}{l}\text { Complete } \\
\text { medium: } 100 \mu \mathrm{L}\end{array}$ \\
\hline & GV493:10 $\mu \mathrm{L}$ & HitransG $A: 4 \mu \mathrm{L}$ & HitransG P:4 $4 \mathrm{~L}$ & \\
\hline $\mathrm{MOI}=100$ & & GV493:10 $\mu \mathrm{L}$ & GV493:10 $\mu \mathrm{L}$ & \\
\hline \multirow{2}{*}{$\begin{array}{l}\text { Group B } \\
5 \times 10^{7}\end{array}$} & $\begin{array}{l}\text { Complete } \\
\text { medium: } 90 \mu \mathrm{L}\end{array}$ & 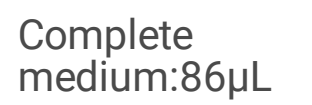 & 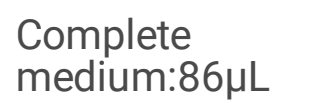 & \\
\hline & GV493:10 $\mu \mathrm{L}$ & HitransG $A: 4 \mu \mathrm{L}$ & HitransG P:4 $4 \mathrm{~L}$ & \\
\hline $\mathrm{MOI}=50$ & & GV493:10 $\mu \mathrm{L}$ & GV493:10 $\mu \mathrm{L}$ & \\
\hline \multicolumn{5}{|l|}{ Group C } \\
\hline \multirow{3}{*}{$\begin{array}{l}1 \times 10^{7} \\
\mathrm{MOI}=10\end{array}$} & $\begin{array}{l}\text { Complete } \\
\text { medium: } 90 \mu \mathrm{L}\end{array}$ & 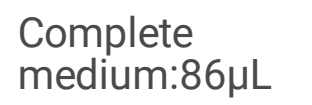 & 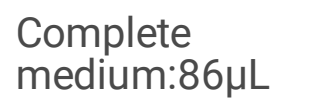 & \\
\hline & GV493:10 $\mu \mathrm{L}$ & HitransG $A: 4 \mu \mathrm{L}$ & HitransG P:4 $4 \mathrm{~L}$ & \\
\hline & & GV493:10 $\mu \mathrm{L}$ & 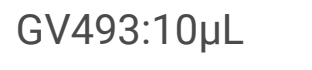 & \\
\hline \multicolumn{5}{|l|}{ Group D } \\
\hline $1 \times 10^{6}$ & $\begin{array}{l}\text { Complete } \\
\text { medium: } 90 \mu \mathrm{L}\end{array}$ & $\begin{array}{l}\text { Complete } \\
\text { medium: } 86 \mu \mathrm{L}\end{array}$ & 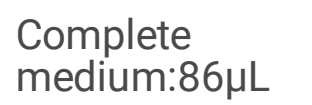 & \\
\hline \multirow{2}{*}{$\mathrm{MOI}=1$} & GV493:10 $\mu \mathrm{L}$ & HitransG A:4 $4 \mathrm{~L}$ & HitransG $P: 4 \mu \mathrm{L}$ & \\
\hline & & GV493:10 $\mu \mathrm{L}$ & GV493:10 $\mu \mathrm{L}$ & \\
\hline
\end{tabular}

hUCMSC complete medium $\square C y a g e n$ Biosciences $\square$ USA $\square$ was used to cultivate hUCMSC. HitransG $\mathrm{P} \otimes \mathrm{Genechem} \nabla$ China $\square$ and Hitrans $G A \otimes G e n e c h e m \llbracket C h i n a \square$ are lentiviral infection enhancer. GV493 was diluted with hUCMSC basal medium $\square$ Cyagen Biosciences $\square U S A \square$ to different titers $\square$ which corresponded to different multiplicity of infection $\square$ MOIV. 


\begin{tabular}{|c|c|c|c|c|}
\hline \multirow[t]{2}{*}{ Group } & virus name & Target sequence & Virus titer & Virus dosage \\
\hline & & & $\otimes T U / m L \bigotimes$ & $\nabla \mu \mathrm{L} \square$ \\
\hline KD1 & LVPSC85384-1 & gcACAGCCAGTAATCGAGTTT & $2 \times 10^{9}$ & 2. 00 \\
\hline KD2 & LVPSC85385-11 & cgGCAAGAAGAGATTAGCAAA & $1 \times 10^{9}$ & 4. 00 \\
\hline KD3 & LVPSC85386-11 & caATCAAATTACAGGCCAATT & $1 \times 10^{9}$ & 4. 00 \\
\hline NC & GW493 & & $1 \times 10^{9}$ & 4. 00 \\
\hline
\end{tabular}

Table 3 The groups and culture conditions of RAFLSs in E-Plate 16 after RA FLSs adherence Groups $₫ n=3 \rrbracket$

Culture condition

QDMEM high sugar medium with $10 \% \mathrm{FBS} \otimes$

Blank control group

$\begin{array}{lc}\text { hUCMSC }^{\text {KD3-Ago2-Exos group }} & 150 \mathrm{ug} / \mathrm{m} \mathrm{hUCMSC}^{\text {KD3-Ago2 }} \text {-Exos } \\ \text { hUCMSC }^{\mathrm{NC}} \text {-Exos group } & 150 \mathrm{ug} / \mathrm{mL} \mathrm{hUCMSC}{ }^{\mathrm{NC}} \text {-Exos }\end{array}$

Table 4 The groups and culture system of RAFLSs in CIM-Plate 16

Group $₫ \mathrm{n}=3 \rrbracket \quad$ upper chamber lower chambers

QSerum-free DMEM high sugar medium $\bigotimes$

Blank control group

hUCMSCKD3-Ago2-Exos
group

hUCMSC $^{\mathrm{NC}}$-Exos group $\quad 150 \mathrm{ug} / \mathrm{mL}^{\text {hUCMSC }} \mathrm{hC}_{\text {-Exos }}$
DMEM high sugar medium with $10 \%$ FBS 
Table $5 \mathrm{a}$ The proliferation of RA FLSs at different time points in each group

\begin{tabular}{|c|c|c|c|}
\hline \multirow[t]{2}{*}{ Group } & \multirow[t]{2}{*}{ Blank control group $\llbracket n=3 \rrbracket$} & \multirow{2}{*}{$\begin{array}{l}\text { hUCMSCKD3-Ago2-Exos } \\
\text { group } \\
\nexists n=3 \rrbracket\end{array}$} & $\begin{array}{l}\text { hUCMSC }{ }^{N C} \text {-Exos group } \\
\bigotimes n=3 \rrbracket\end{array}$ \\
\hline & & & \\
\hline
\end{tabular}

Time point

\begin{tabular}{|c|c|c|c|c|}
\hline $10 \mathrm{~h}$ & 1. $5394 \pm 0 . \quad 0724222$ & 1. $37 \pm 0.075$ & 538 & $\begin{array}{l}\text { 1. } 3084333 \pm 0 . \\
0346791\end{array}$ \\
\hline $20 \mathrm{~h}$ & $\begin{array}{l}\text { 1. } 5847667 \pm 0 . \\
0686025\end{array}$ & 1. $3421667 \pm 0$. & 0810739 & $\begin{array}{l}\text { 1. } 2312333 \pm 0 . \\
0228715\end{array}$ \\
\hline $30 \mathrm{~h}$ & 1. $5327 \pm 0 . \quad 0559024$ & 1. $1817667 \pm 0$. & 0421836 & $\begin{array}{l}\text { 1. } 0848333 \pm 0 . \\
0167804\end{array}$ \\
\hline $40 \mathrm{~h}$ & $\begin{array}{l}\text { 1. } 8286333 \pm 0 . \\
0687184\end{array}$ & 1. $4050333 \pm 0$. & 0777613 & $\begin{array}{l}\text { 1. } 2751333 \pm 0 . \\
0329749\end{array}$ \\
\hline
\end{tabular}

Table 5b The migration of RA FLSs at different time points in each group

\begin{tabular}{|c|c|c|c|}
\hline \multirow[t]{2}{*}{ Group } & \multirow[t]{2}{*}{ Blank control group $₫ n=3 \rrbracket$} & $\begin{array}{l}\text { hUCMSCKD3-Ago2-Exos } \\
\text { group }\end{array}$ & $\begin{array}{l}\text { hUCMSC }{ }^{N C} \text {-Exos group } \\
\rrbracket n=3 \rrbracket\end{array}$ \\
\hline & & $\llbracket n=3 \rrbracket$ & \\
\hline
\end{tabular}

Time point

$10 \mathrm{~h}$

1. $2837 \pm 0$. 0145506

1. $2204667 \pm 0$.

0461880

1. $0878000 \pm 0$.

0775234

$20 \mathrm{~h}$

1. $098 \pm 0.0401598$

1. $0769 \pm 0$. 0270217

0. $9862667 \pm 0$.

0544596

$30 \mathrm{~h}$

1. $2325333 \pm 0$.

0631646

1. $2112 \pm 0$. 0409297

1. $1449667 \pm 0$.

0442838

$40 \mathrm{~h}$

1. $3565 \pm 0$. 0780123

1. $3427667 \pm 0$. 041502

1. $2802333 \pm 0$.

0581369

\section{Figures}




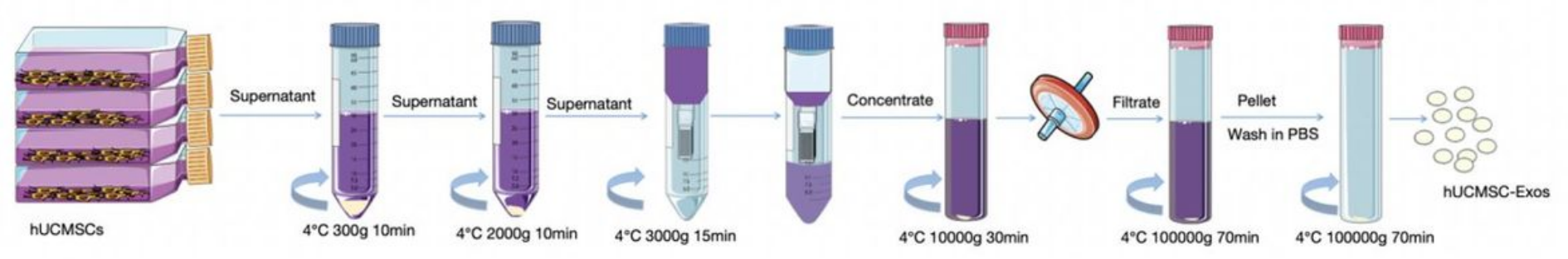

\section{Figure 1}

The supernatant of hUCMSCs was sequentially removed from dead cells and cell debris under different centrifugation conditions. After that \the supernatant was concentrated with an ultrafiltration tube and filtered with $0.22 \mu \mathrm{m}$ filter membranes. Then the higher centrifugation conditions removed smaller debris. Finallyヌultra-high-speed centrifugation to pellet and wash exosomes.

a

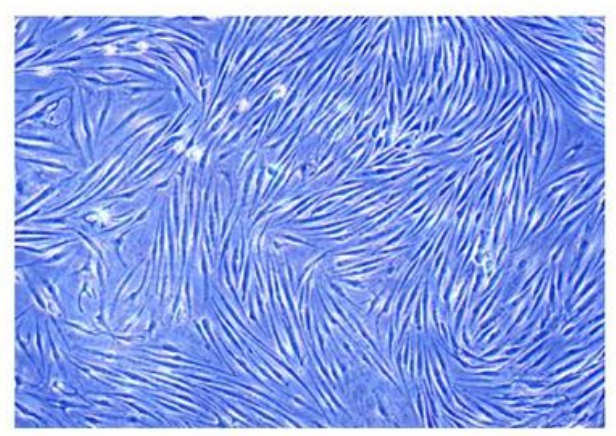

b
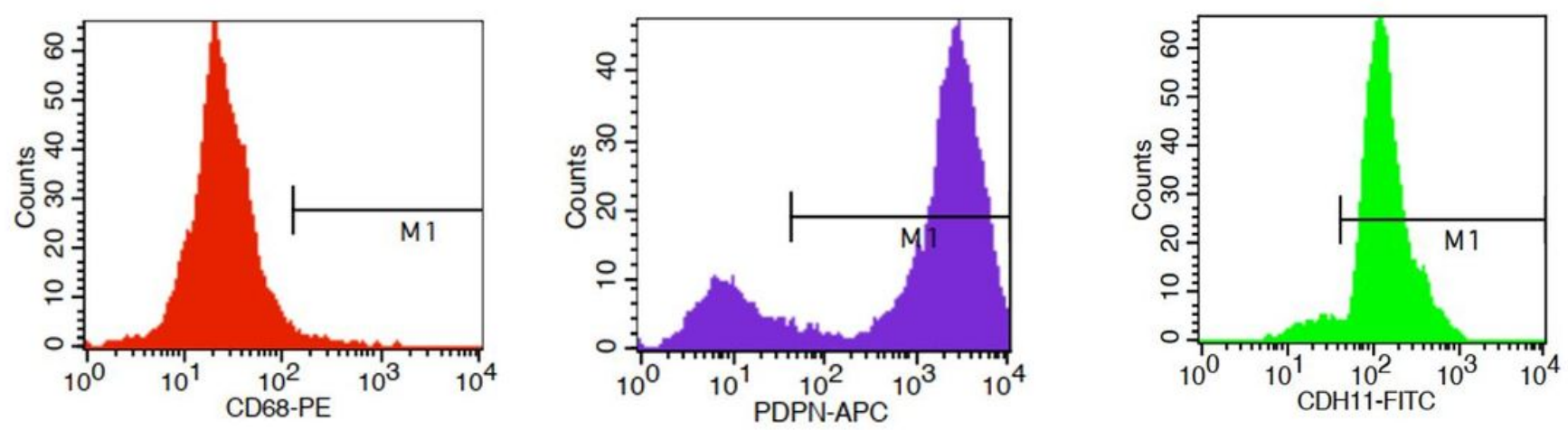

\begin{tabular}{rrrr} 
Marker & Left, Right & Events & $\%$ Gated \\
\hline All & 1,9910 & 8744 & 100.00 \\
M1 & 126,9910 & 189 & 2.16
\end{tabular}

\begin{tabular}{rrrr} 
Marker & Left, Right & Events & $\%$ Gated \\
\hline All & 1,9910 & 8744 & 100.00 \\
M1 & 43,9910 & 7144 & 81.70
\end{tabular}

\begin{tabular}{rrrr} 
Marker & Left, Right & Events & $\%$ Gated \\
\hline All & 1,9910 & 8134 & 100.00 \\
M1 & 42,9910 & 7647 & 94.01
\end{tabular}

\section{Figure 2}

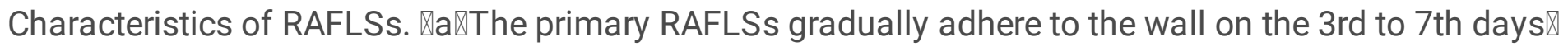
and are fully grown and passable at about 18-25 days. They displayed a fibroblast-like morphology. When the growth density is $\geq 70 \%$ they will appear "vortex". 区bखRAFLSs express PDPN and CDH-11 positively on the surface $\$ and negatively express CD68 
a

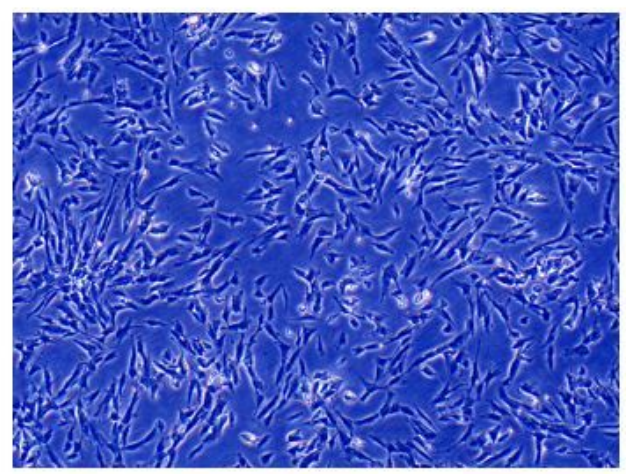

b

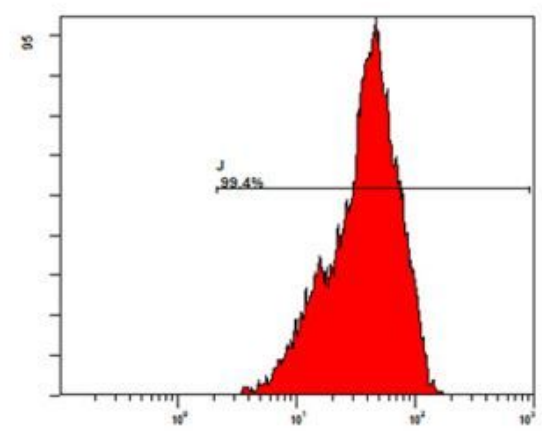

CD105

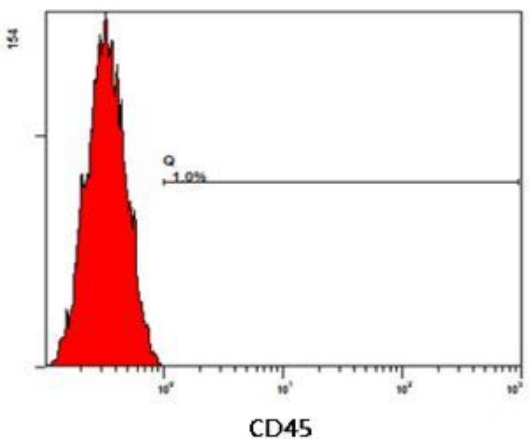

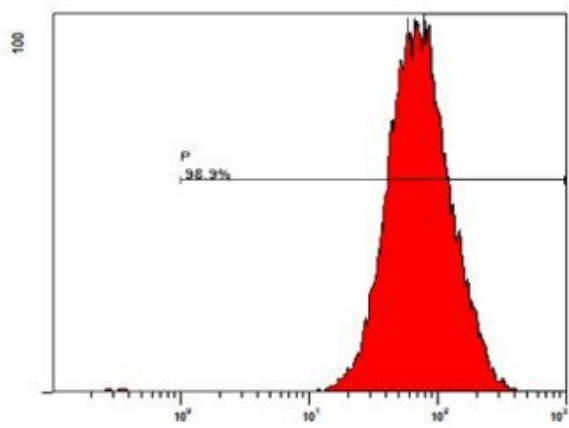

CD73

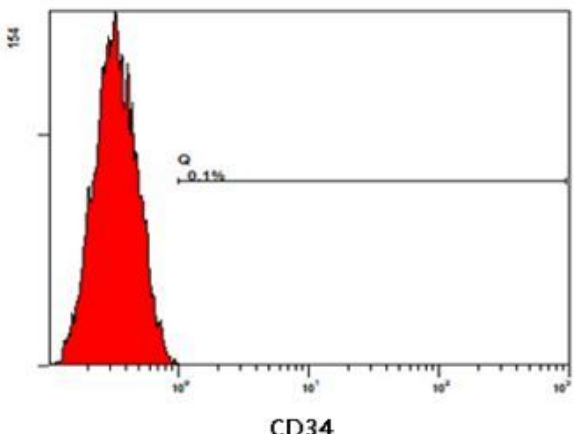

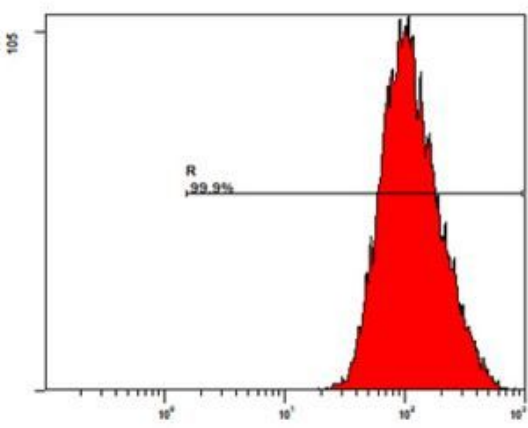

CD90

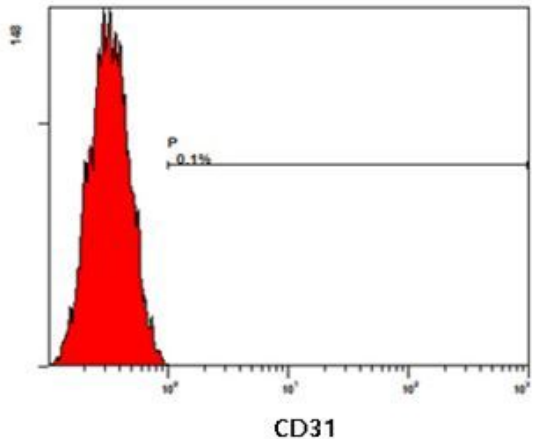

Figure 3

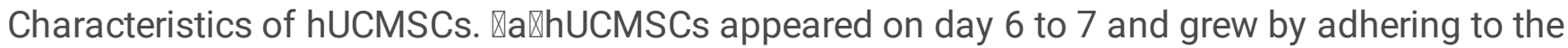
substratum with a long spindle-like morphology . The growth density of HUCMSCs reached $80 \%$ in about 16-20 days. $\mathrm{P} 2$ hUCMSCs grow faster and can be subcultured in about 5 days. खbखFCM analysis of

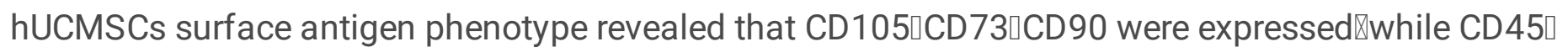
CD34匹CD31 were not . 


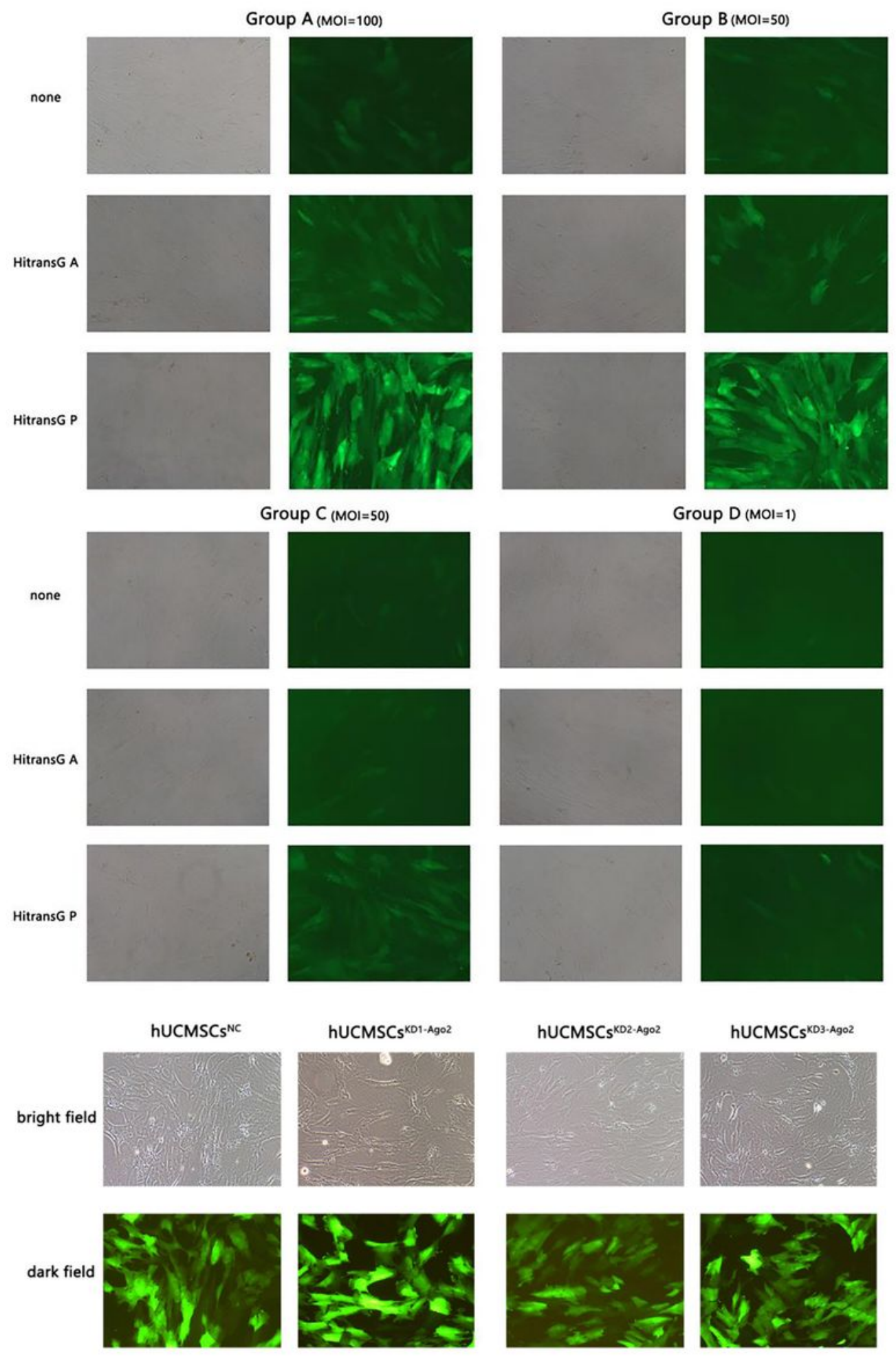

\section{Figure 4}

a The morphology and proliferation of the transfected hUCMSCs were monitored under bright-field and dark field microscopy冈and they are normal. Adding HiTransG P to each group can enhance the

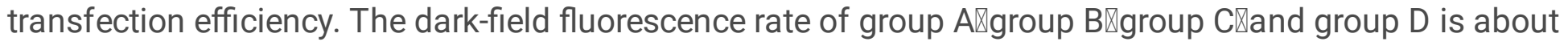
$100 \% \varangle 90 \% \varangle 60 \% \otimes 20 \% \otimes$ The best fluorescence rate is $80 \%$ obviously $₫$ The best $\mathrm{MOI}$ is between $10-50$. We finally choose $\mathrm{MOI}=20$ and HiTransG P to transfect hUCMSCs. Expression of the green fluorescence 
protein $₫$ GFP $₫$ is observed as green colored hUCMSCs. b hUCMSCs morphology was monitored under bright-field microscopy as well as dark field microscopy . GV493 with or without siRNA targets could successfully transfected hUCMSCs囚Expression of the GFP is observed as green colored hUCMSCs.

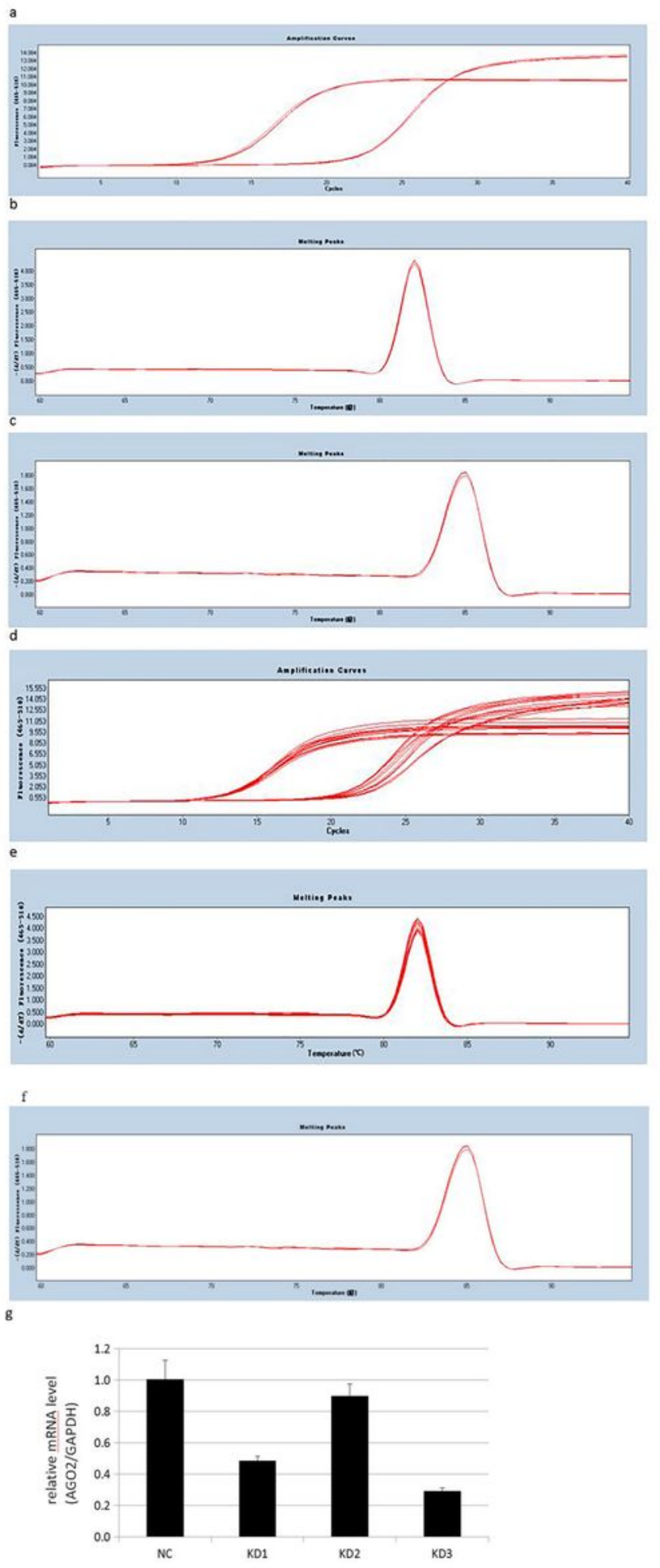

Figure 5

RT-PCR results of Ago2 in hUCMSCs \hUCMSCNC and hUCMSCKD-Ago2】a囚Amplification curves of GAPDH and Ago2 genes in hUCMSCs. खb区Dissolution curve of Ago2 in hUCMSCs. 『c区Dissolution curve of 
GAPDH in hUCMSCs. 『d \Amplification curves of GAPDH and Ago2 in hUCMSCNC and hUCMSCKD-Ago2.

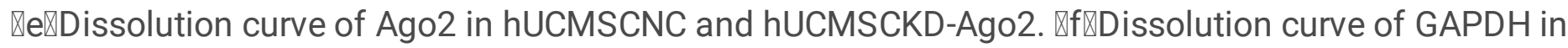
hUCMSCNC and hUCMSCKD-Ago2. 『g『Knockdown efficiency of Ago2 in hUCMSCNC and hUCMSCKDAgo2.

a

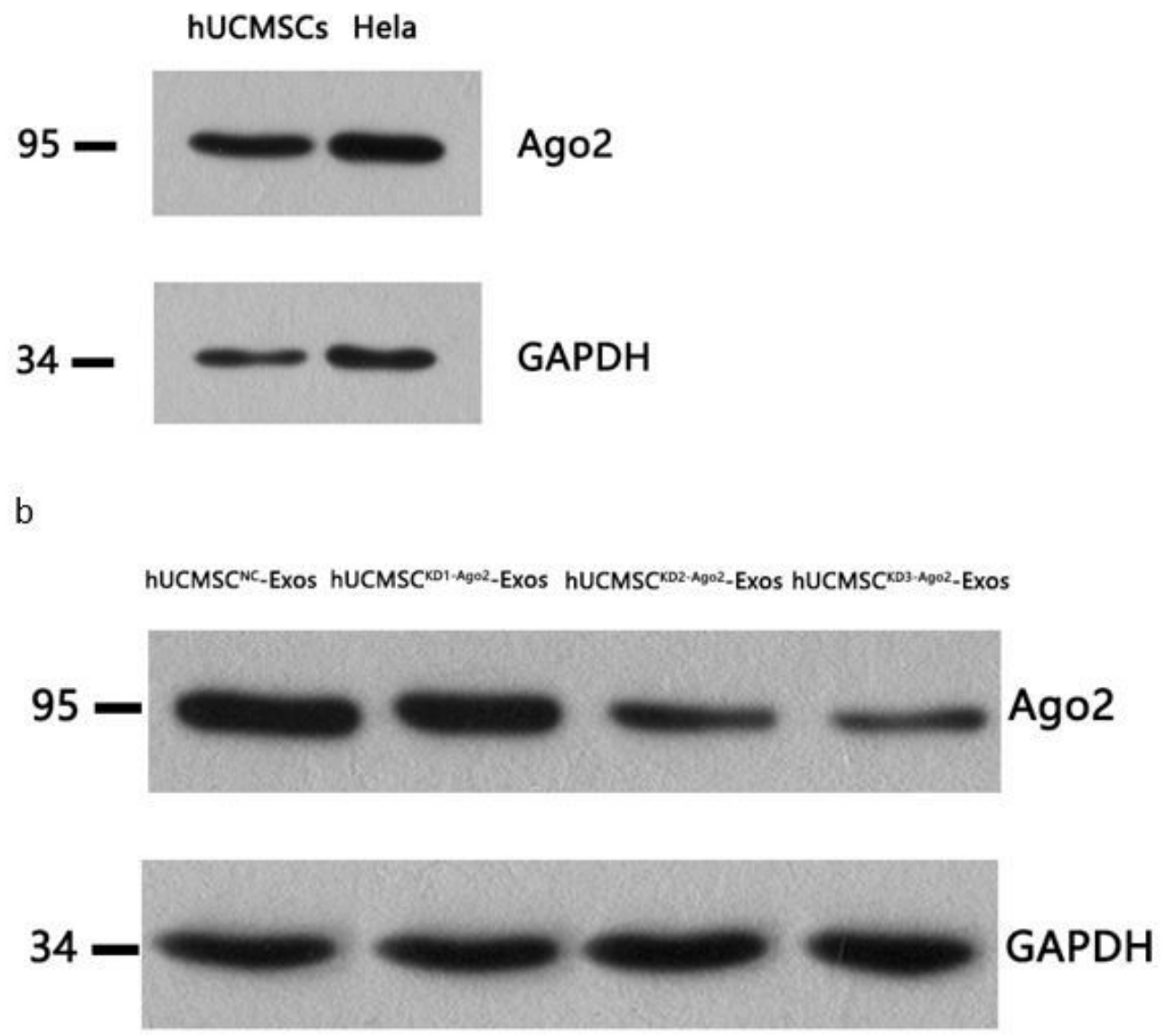

c

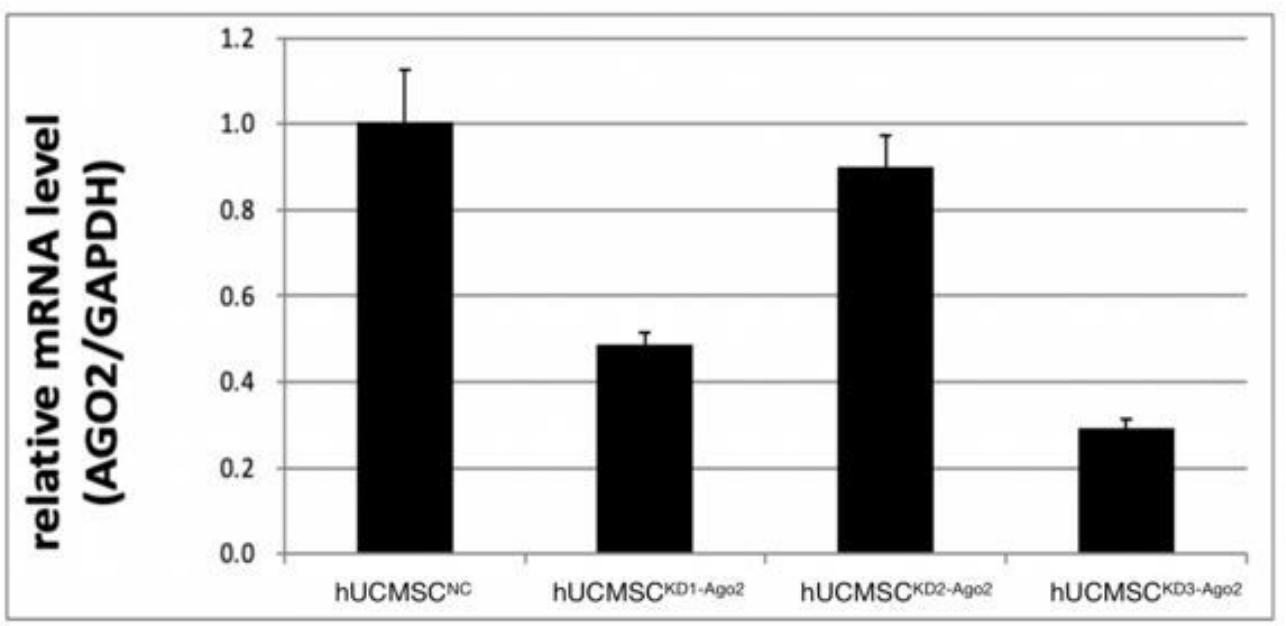

Figure 6 


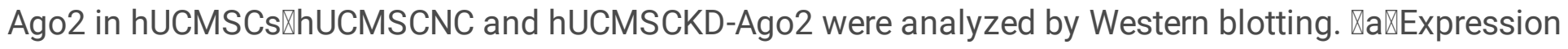
of Ago2 in hUCMSCs. 『b囚Expression of Ago2 in hUCMSCNC and hUCMSCKD-Ago2. 『c 『Ago2 band gray value of hUCMSCNC and hUCMSCKD-Ago2.
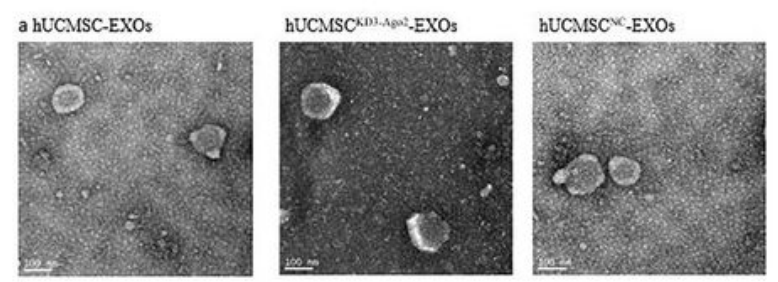

b
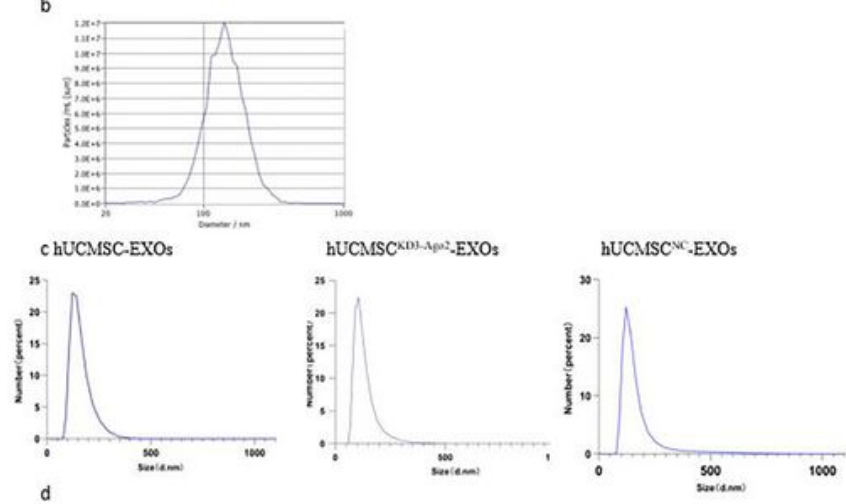

hUCMSC-Exos hUCMSC ${ }^{-}$-Exos hUCMSCorner-Exos

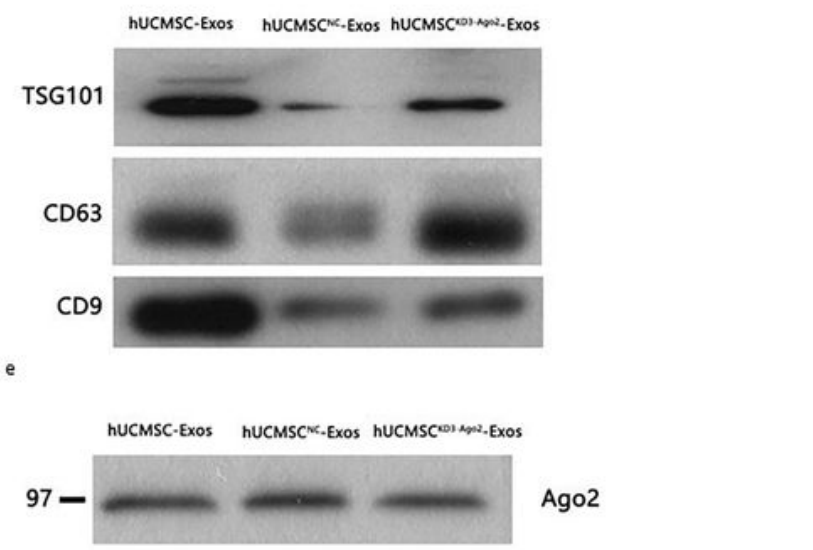

$\mathrm{F}$

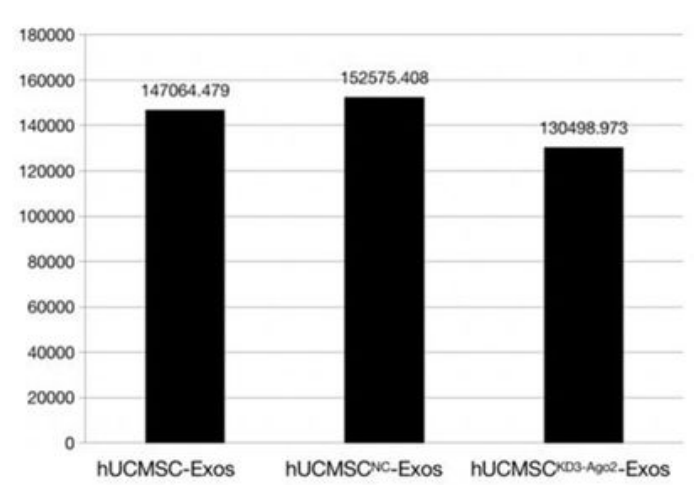

Figure 7

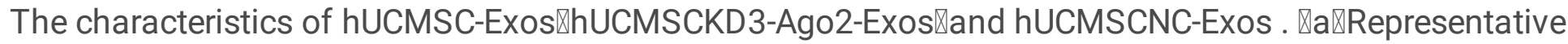
transmission electron microscopy $₫ T E M \otimes$ analysis from hUCMSCs $₫$ hUCMSCNC and hUCMSCKD3-Ago2. 


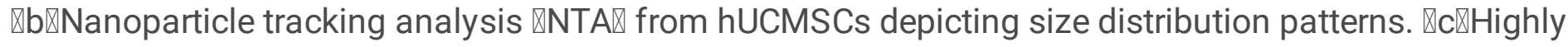
sensitive nanoparticle size analysis from hUCMSC-Exos $\llbracket$ hUCMSCKD-Ago2-Exos》and hUCMSCNC-Exos

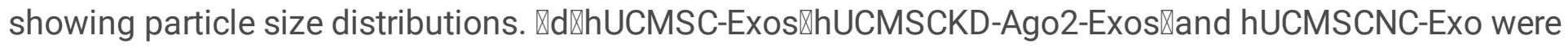
analyzed by Western blotting using exosome markers. Representative images were shown for CD9『CD63

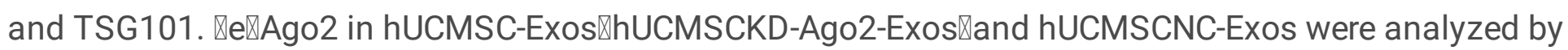

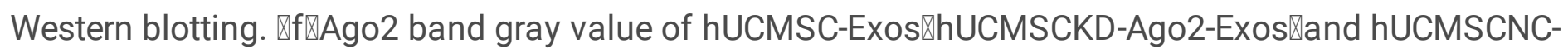
Exos.

a

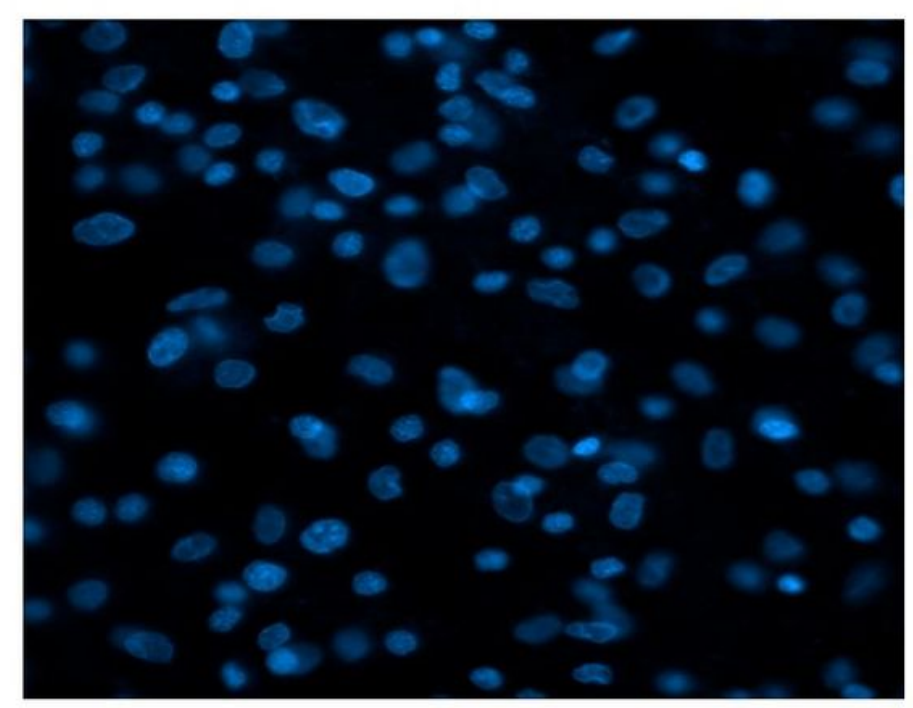

b

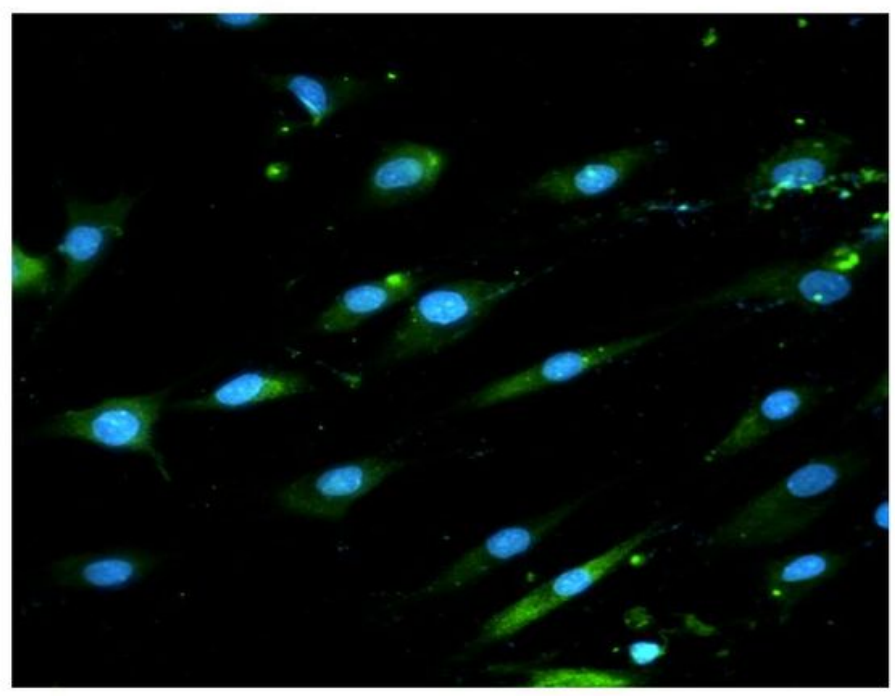

Figure 8

The uptake of RNA in hUCMSC-Exos by RAFLSs. 『a冈The nucleus of RA FLS stained by Hoechst 33342. \b囚The blue-stained nucleus of RA FLSs were accumulated by green fluorescencet $₫$ which is hUCMSCExos with green fluorescent-labeled RNA. 
a

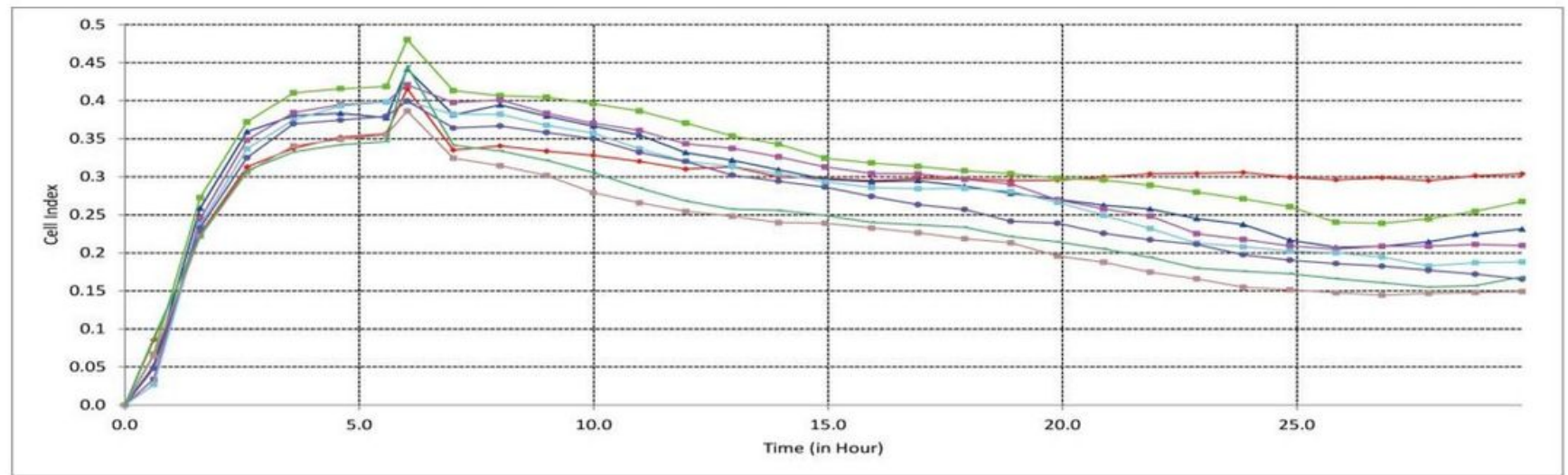

o ug/ml

$10 \mathrm{ug} / \mathrm{ml}$

$30 \mathrm{ug} / \mathrm{ml}$

$60 \mathrm{ug} / \mathrm{ml}$

$90 \mathrm{ug} / \mathrm{ml}$

$120 \mathrm{ug} / \mathrm{ml}$

$150 \mathrm{ug} / \mathrm{ml}$

$180 \mathrm{ug} / \mathrm{m}$

b

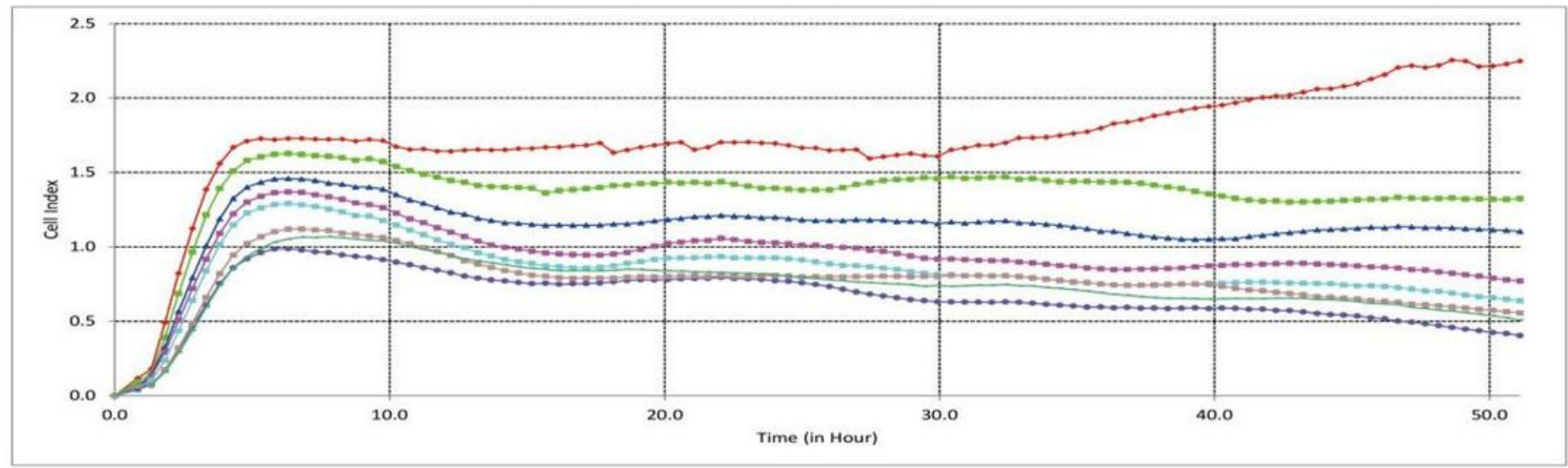

o ug/m!

\section{Figure 9}

The effect of hUCMSC-Exos on the RA FLSs. 『a冈After different concentrations of hUCMSC-Exos interfere

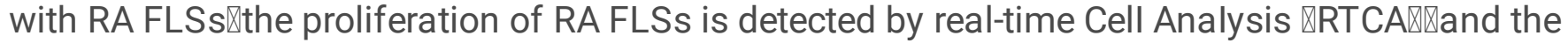
$150 \mathrm{ug} / \mathrm{mL}$ hUCMSC-Exos inhibited the proliferation of RAFLSs most strongly. खbखCultured RA FLS in serum-free medium with different concentrations of hUCMSC-Exos in the upper chamber of CIM-Plate 16区 added $15 \%$ FBS into the lower chamber of RTCA囚and RTCA detected the migration of RA FLS $₫$ and the $120 \mathrm{ug} / \mathrm{mL}$ hUCMSC-Exos inhibited the proliferation of RAFLSs most strongly. 


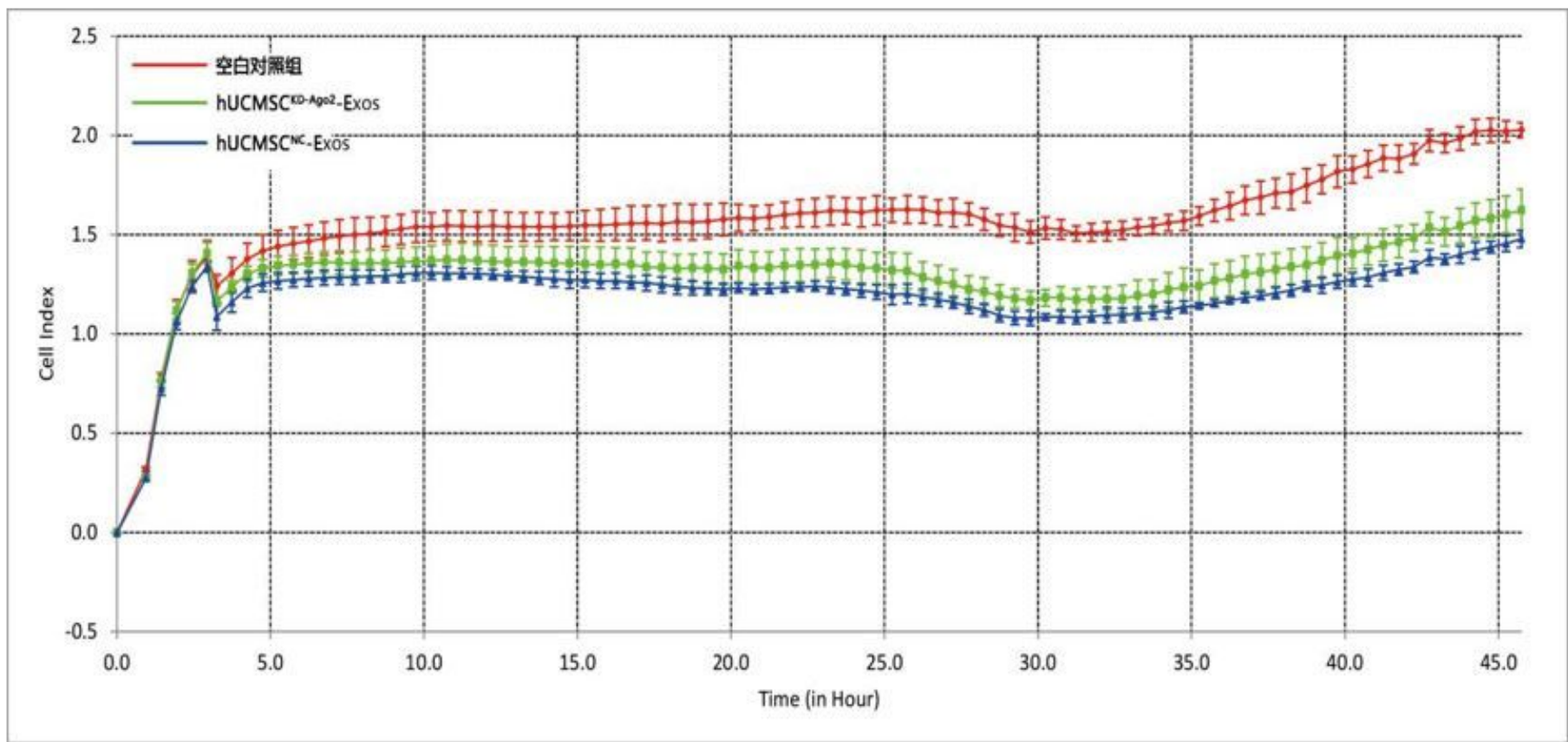

b

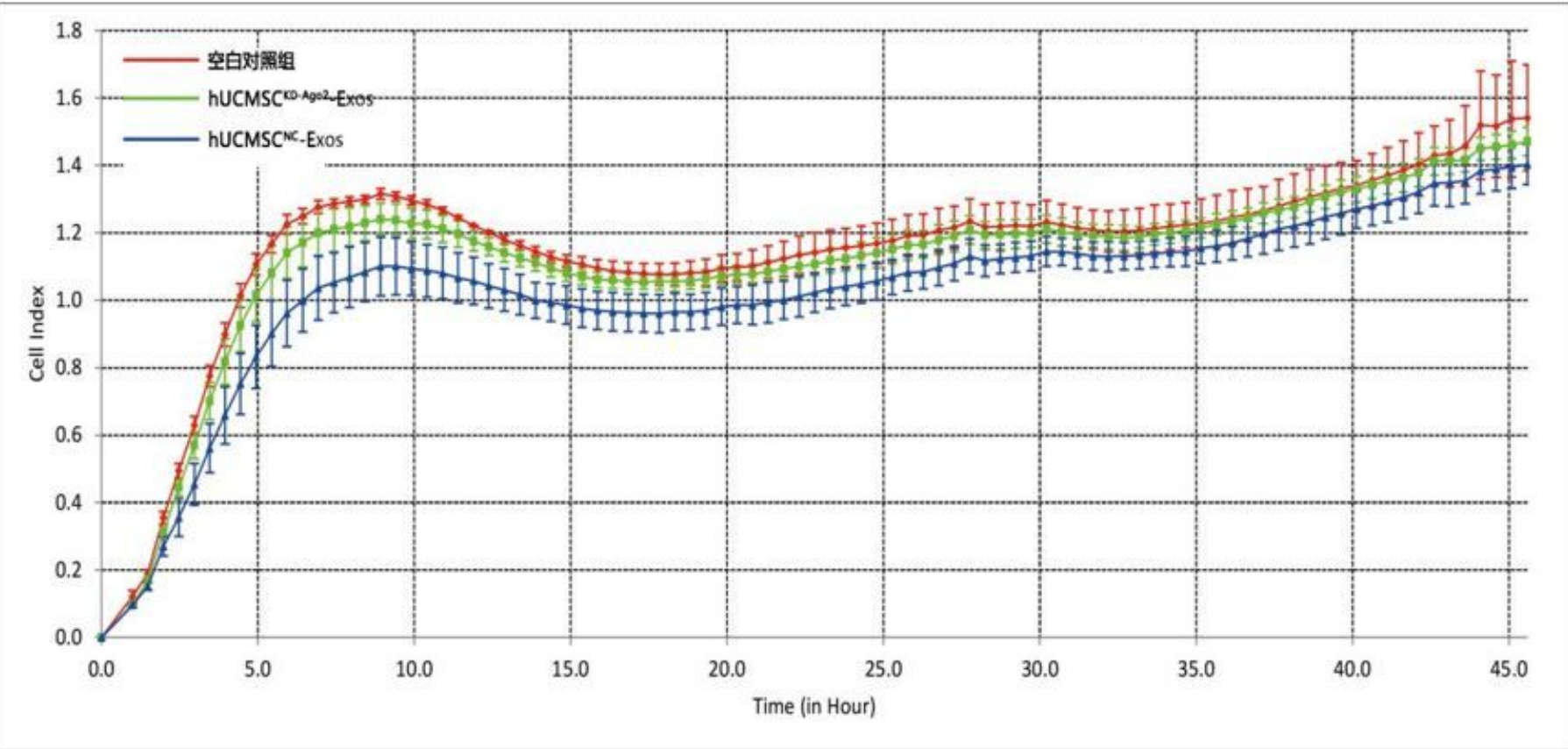

\section{Figure 10}

The effect of hUCMSC-Exos miRNA on the proliferation and migration of RAFLSs. 『a冈The RA FLS proliferation curve showed that compared with the blank control『both the hUCMSCKD3-Ago2-Exos and hUCMSCNC-Exos groups inhibited the proliferation of RAFLSs囚particularly hUCMSCNC-Exos had the

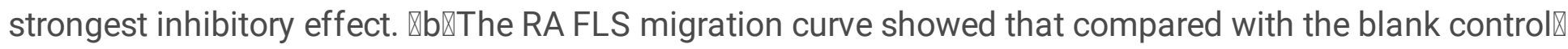

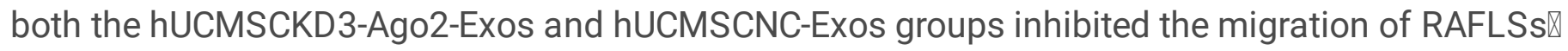
particularly hUCMSCNC-Exos had the strongest inhibitory effect. 